\title{
Characteristics of new star cluster candidates in the Cygnus area
}

\author{
J.-M. Le Duigou and J. Knödlseder
}

\author{
Centre d'Étude Spatiale des Rayonnements, CNRS/UPS, BP 4346, 31028 Toulouse Cedex 4, France \\ e-mail: Jean-Michel.LeDuigou@cnes.fr; knodlseder@cesr.fr
}

Received 12 April 2002 / Accepted 6 June 2002

\begin{abstract}
The morphology and stellar content of 22 clusters in the Cygnus area has been determined using 2MASS infrared observations in the $J$ and $K$ bands. 7 of the clusters are well-known objects and our results are in good agreement with previous work. 12 objects are recently-discovered cluster candidates (Dutra \& Bica 2001) and 3 are new cluster candidates. Adopting distance estimates from the literature or by considering cluster counterparts, we derive a consistent set of parameters suitable for analysis and comparison: center coordinates, radius, stellar population, IMF slope and mass range. We find a mean IMF slope of $\Gamma=-1.30$ for the known clusters and $\Gamma=-1.40$ for the others. Infrared data having better completeness, we derive larger stellar contents and cluster masses than previous works. An important fraction of the cluster stellar population in Cygnus is hidden in very obscured areas. A clear tendency towards mass segregation appears for most of the clusters. Some of the new clusters seem to be quite massive, probably hosting very massive stars at the center. They are privileged targets for more detailed investigations using infrared spectroscopy.
\end{abstract}

Key words. stars: early-type - ISM:dust: extinction - open clusters and associations: individual: Cygnus Galaxy: stellar content

\section{Introduction}

The Cygnus area includes a lot of interesting young objects: five $\mathrm{OB}$ associations, numerous young open clusters, tens of compact HII regions and star nurseries. Its relative proximity of 1-2 kpc makes it one of the most exciting regions for studies of the star formation process and efficiency, the interaction of massive stars with the interstellar medium, the initial mass function and stellar nucleosynthesis.

Unfortunately, the Cygnus region is a rather complex area. It is usually accepted to be the local arm seen tangentially so that many structures superpose in the line of sight. Furthermore, and most important, heavy absorption with complex patterns severely affects the surveys performed in the visible and $U V$ range. This situation probably leads to an underestimation of the star population, possibly even missing some OB stars in the most obscured areas. Many star-forming regions, probably hosting very young clusters, remained unresolved although the presence of massive stars is deduced from the ionization flux measurements in the infrared (Odenwald et al. 1986; Odenwald \& Schwartz 1993). The recent availability of the 2MASS survey provides an excellent opportunity to better characterize clusters in the Cygnus area, the infrared data being far less sensitive to absorption.

Send offprint requests to: J.-M. Le Duigou, e-mail: Jean-Michel.LeDuigou@cnes.fr
The first attempt concentrated on the most massive object in the area: the Cygnus OB2 compact association (Knödlseder 2000 , hereafter referred to as Paper I). The combination of infrared photometry with a star count method based on photometric selection and morphology analysis proved to be a very efficient tool to analyze the structure. It demonstrated that previous studies were biased by the absorbing patterns and that they considerably underestimated the stellar content. The conclusion was to reclassify this object as a young globular cluster.

Encouraged by this success, we decided to generalize the process in order to characterize clusters located in the Cygnus area and search for new candidates that are hidden in the visible by interstellar dust clouds. We could derive a homogeneous set of parameters that is suitable for comparative analysis. We will first briefly describe our method, then its validation by application to some well-known clusters and comparison to published results. Then we will present the results for the 15 previously unknown or recently discovered clusters. Finally, a discussion of the main results leads to our conclusions.

\section{Description of the method}

\subsection{Cluster identification}

The studied area was chosen so as to cover the five main OB associations in the Cygnus area (Garmany \& Stencel 1992) with comfortable margins. The selected window is defined by 


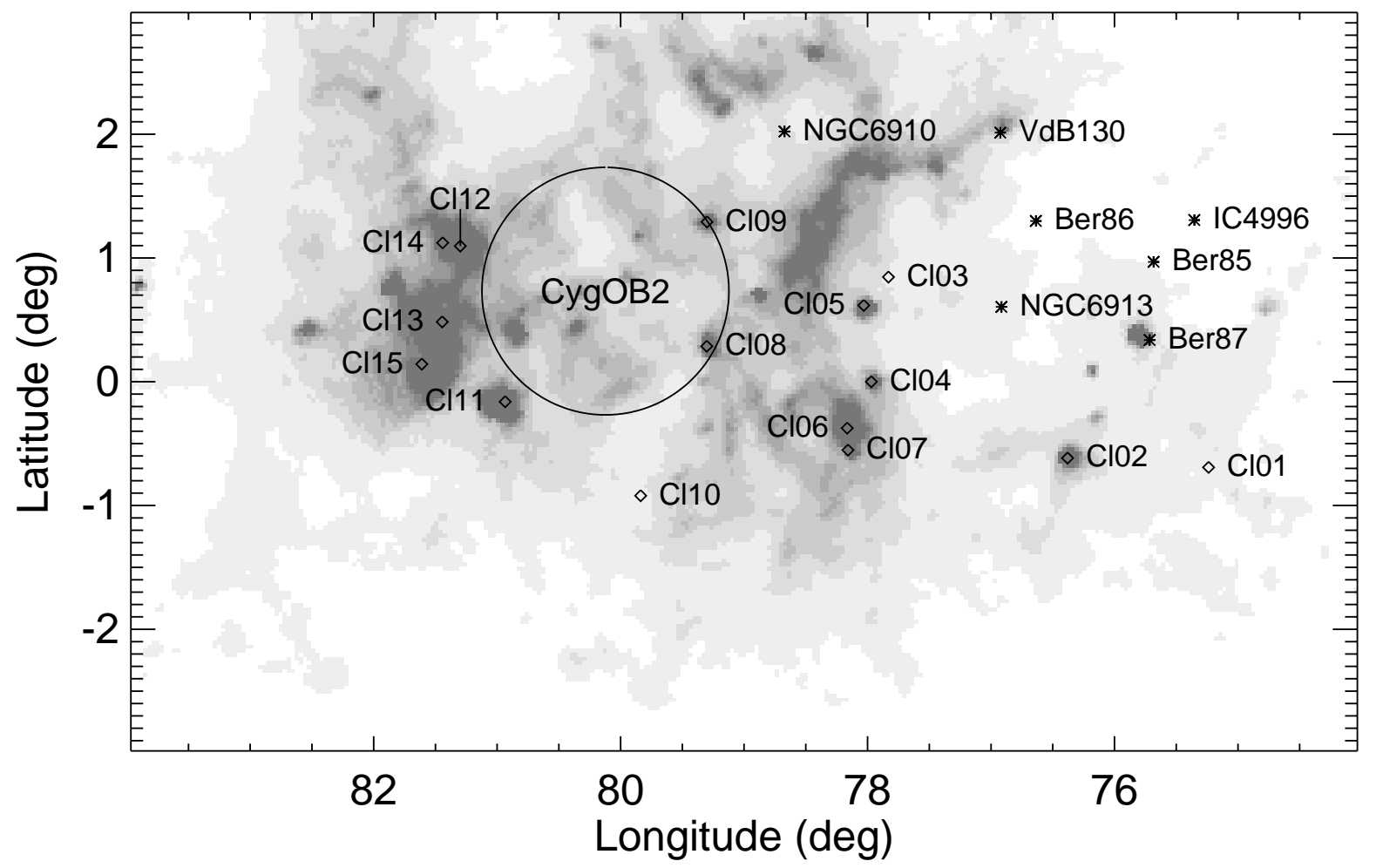

Fig. 1. Map of the open clusters in the Cygnus area. The clusters are superimposed on the $100 \mu \mathrm{m}$ IRAS map which traces the distribution of dust. Asterisks correspond to previously known clusters, open diamonds correspond to new cluster candidates. The circle represents the spatial extension of Cygnus OB2.

galactic longitude $70^{\circ} \leq l \leq 83^{\circ}$ and latitude $-3^{\circ} \leq b \leq 7^{\circ}$. The filtering of the 2MASS second edition PSC lead to 5119088 stars with celestial coordinates and $J, H$, $K$ magnitudes. We selected all stars regardless of the photometric quality. Less than $0.15 \%$ of the sample is quoted as stars with uncertain photometry or with saturation. $4.2 \%$ are affected by nearby bright star diffraction spikes, but we masked these zones, as will be seen later, so the induced errors are negligible compared to distance uncertainties and heavy reddening effects.

In a second step, using star density maps with a $2 \times$ $2^{\prime}$ bin size, we scanned our dataset to detect apparent stellar concentrations. This procedure resulted in a list of cluster candidates that could be true clusters, superposition effects of field stars, or effects of the absorption patterns. Repeating the operation with stars only in a given spectral type range and/or having estimated absorption in a given range helped to distinguish between these categories. Some of the cluster candidates corresponded to known clusters and were kept in the list to be analyzed for comparison with existing data. The other candidates were analyzed individually. They were supposed to be true clusters only if they had a clear globular morphology and if one could detect a main sequence distribution in the color magnitude diagram (CMD). This process lead to a list of 22 clusters. Their location is illustrated in Fig. 1, superimposed on the $100 \mu \mathrm{m}$ IRAS data that traces the interstellar dust. The spatial coincidence of almost all the new cluster candidates (diamonds) with very heavily obscured zones, which are also HII regions in many cases, is striking. It is also very interesting to note that most of them are located at the border of the Cygnus OB2 association. The previously known clusters (asterisks) are located in less obscured regions.

Some clusters were detected but were not analyzed for the following reasons: NGC 6871 has a very bright star in the foreground very close to the cluster center, most of the Dolidze clusters (Dolidze 1961) have too few stars to be analyzed by our method and the Cyg OB2 compact association has been previously characterized.

The characterization of each cluster was an iterative process starting with an initial set of parameters. First estimates of the center $\alpha, \delta$ and the diameter $\phi$ were derived from the process described above. A square field of two times the size of the cluster was selected to determine the field star densities.

\subsection{Cluster characterization}

\subsubsection{Completeness}

We first estimated the completeness magnitude limits for each analyzed cluster in the three bands by plotting differential star count distributions versus apparent magnitude. For this process we used all stars in the square of size $a$ around the selected cluster. The turn-off points in these curves give us two limits: $J_{\lim }$ and $K_{\lim }$. A more precise estimation is not needed because we do not try in this work to study the faint star population. We just want to ensure that our main range of interest $(\mathrm{O}-\mathrm{F} 3)$ is complete. 


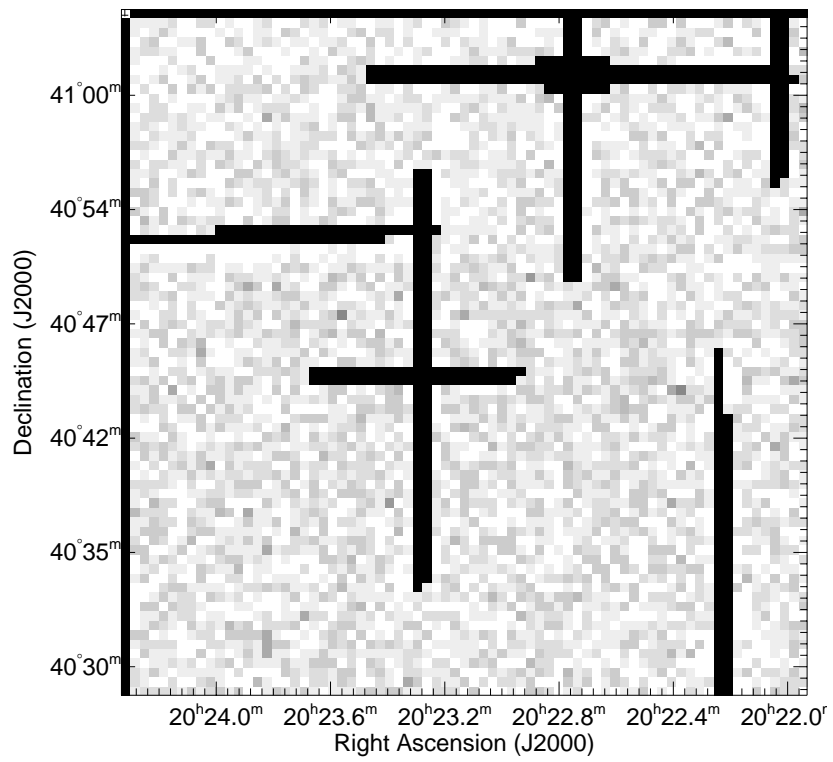

Fig. 2. Mask of density map for NGC 6910. The bin size is here $0.5 \times 0.5^{\prime}$. The black crosses are the masked areas where photometry is affected by foreground bright stars.

\subsubsection{Morphology, radial profiles and stellar content}

In a second step we studied the cluster morphology by using star density maps. The raw star density maps are simply a binning of a given square area around a cluster. The adopted bin size for cluster characterization was $0.25 \times 0.25^{\prime}$. The raw density maps, using all stars without any filtering, are used to identify diffraction spikes of very bright foreground stars. The corresponding areas appear as blank crosses in the maps. We detect them using a simple algorithm which supposes the crosses to be aligned with the celestial coordinates axes. It detects the lines of consecutive pixels with zero or near-zero star density in both directions. These areas define a mask of excluded regions, as shown by Fig. 2 .

Filtered density maps are calculated to improve the cluster characterization. Assuming DM and $A_{K}$ are known for the cluster, the filtering uses a spectral type range specification and an allowed absorption range to better extract the cluster morphology from the field. This photometric selection is fully detailed in Sect. 2.2.3. Spectral types are related to $K$ absolute magnitude using Table A.1 of Paper I. Smoothed density maps are then calculated to remove statistical fluctuations. During the process, the star density in the masked areas is interpolated from the adjacent values. Hence, the areas of missing stars due to bright star diffraction spikes are corrected for. Furthermore, an estimation of the background gradient using a least square method is performed. This gradient is subtracted from the map to obtain clean maps as illustrated by Fig. 3. In the left map, there is no absorption selection and the cluster structure hardly appears. The right map was plotted using only stars with absorption in the $K$ band lower than $0.7^{\mathrm{m}}$, corresponding to the known absorption range of the cluster. The morphology of NGC 6910 appears clearly here, showing the efficiency of the selection method to extract the cluster morphology. Note also how the algorithm smoothed the area affected by bright stars imprints removing their effect on the morphology.

In order to quantify the star population of the cluster and to estimate the associated errors, we determined star density radial profiles. The studied area is divided into 19 concentric annuli based on regular steps from 0 to $a$. For each annulus, we calculate a mean radius $R_{i}$ on the basis of equal area interpolation. The corresponding surface $S_{i}$ is the surface of the annulus $S_{i, 0}$ minus its intersection with the mask area. The star population $N_{i}$ is determined from the finely binned star density maps by integration over $S_{i}$. The estimated gradient is removed. Then, the star density $n_{i}$ and the associated error $\epsilon_{i}$, assuming a Poisson law error in the star counts, are derived simply by $n_{i}=N_{i} / S_{i}$ and $\epsilon_{i}=\sqrt{N_{i}} / S_{i}$.

Using cumulated star counts versus radius, obtained from summation of the star density after field density removal, one can first derive an estimation of the radius containing $90 \%$ or $50 \%$ of the star population $\left(r_{90}\right.$ and $\left.r_{50}\right)$. Then, the radial profile (see Fig. 4) is fitted using a King profile (King 1962) on top of a constant field star density:

$f(r)=F S D+k\left\{\left[1+\left(r / r_{\mathrm{c}}\right)^{2}\right]^{-1 / 2}-\left[1+\left(r_{\mathrm{t}} / r_{\mathrm{c}}\right)^{2}\right]^{-1 / 2}\right\}^{2}$.

For a given center, the core radius $r_{\mathrm{c}}$ is usually well defined and insensitive to details of the stellar selection. The tidal radius $r_{\mathrm{t}}$, however, is poorly determined, hence we will not use it as a relevant parameter for cluster characterization. The main objective of this fitting is the precise estimation of the field star density FSD. The fitting process also gives the associated error $\epsilon_{F S D}$. Using these results, one can estimate the stellar cluster population and the associated error, adding quadratically the various error sources:

$$
\begin{aligned}
& N=\sum_{i=0}^{i_{\max }} n_{i} \times S_{i, 0}-F S D \times \pi r_{\max }^{2} \\
& \epsilon_{N}=\sqrt{N+\epsilon_{\mathrm{FSD}}^{2} \times \pi r_{\text {max }}^{2}} \quad r_{\text {max }}=\phi / 2 .
\end{aligned}
$$

We also determined the cluster center by minimizing its radial extent. We choose to perform this analysis with the spectral range $\mathrm{O}$ to $\mathrm{F} 3$ and within the optimum absorption range as deduced from the CMD analysis (see below).

\subsubsection{Color magnitude diagram (CMD)}

The morphological analysis was complemented with a photometric analysis using color magnitude diagrams in the $K$, $J-K$ domain. We used the same stellar calibration as in the study of Cygnus OB2 (see Appendix A in Paper I and references therein). It defines relations between the spectral type, $M_{V},(V-K)_{0}, M_{K},(J-K)_{0}, \tilde{K}$ (see definition below). Because the objects we concentrate upon are very young, most of the stars are still on the main sequence and we may use the class V luminosity calibration only. This is a major assumption in this study.

As shown in Paper I, all member stars of an open cluster with identical intrinsic magnitude but different extinction lie in the $K, J-K$ domain on a line of constant slope given by:

$K=\tilde{K}+D M+R_{K} \times(J-K)$ 

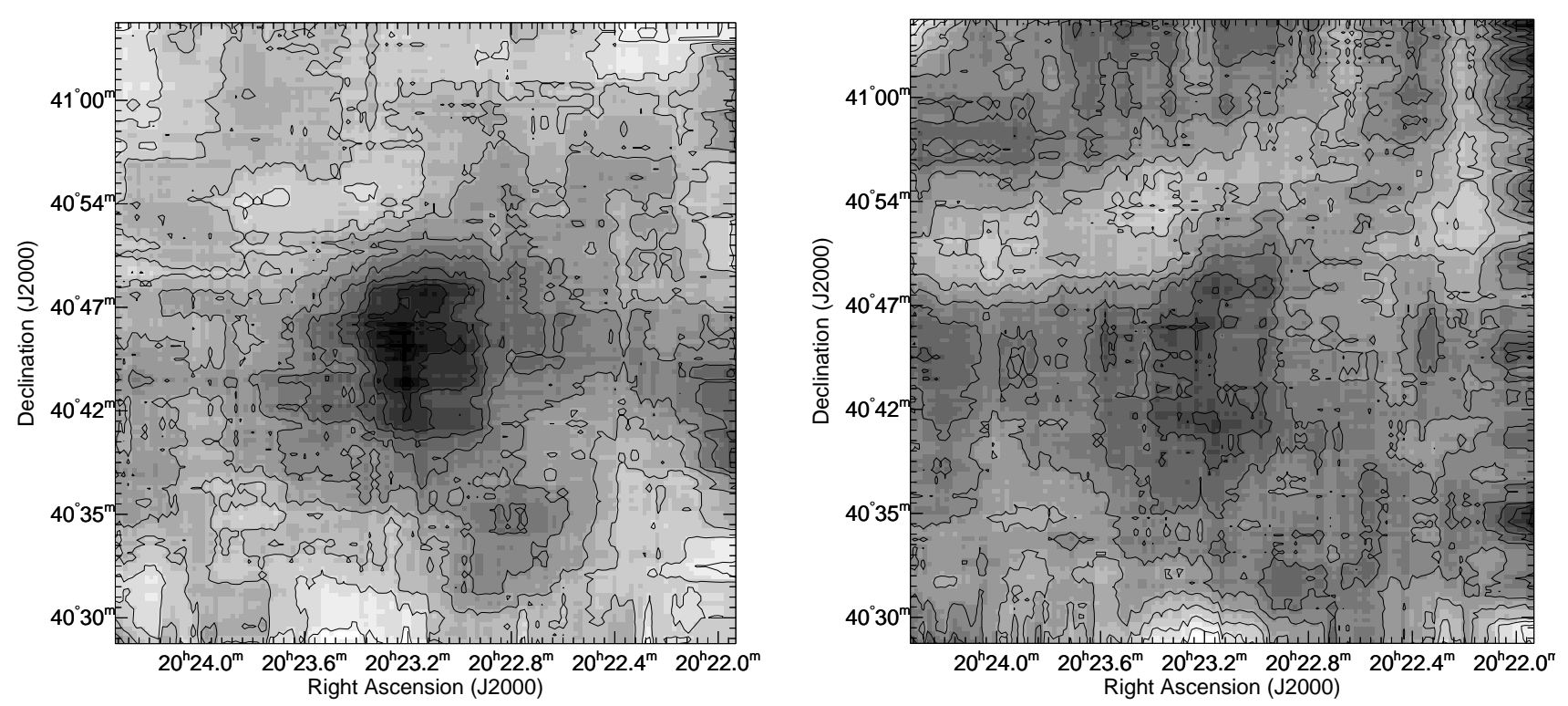

Fig. 3. Smoothed density maps for NGC 6910 using a $4 \times 4^{\prime}$ smoothing bin size and O to F3 stars. On the left, no selection on $A_{K}$ is applied, while on the right $A_{K}<0.7^{\mathrm{m}}$.

where $\tilde{K}=M_{K}-R_{K} \times(J-K)_{0}$ and $A_{K}=R_{K} \times E(J-K)$. $\mathrm{DM}$ is the assumed distance modulus of the cluster. $R_{K}$ is the reddening slope in the $K$ band. Results have low sensitivity to this parameter and we adopted $R_{K}=0.66$ throughout this work (Rieke \& Lebofsky 1985).

Using a linear fit of the relation between $M_{K}$ and $\tilde{K}$, one can derive an approximate relation to estimate the extinction $A_{K}$ (see Paper I for details) :

$A_{K} \approx \frac{K+0.057-17.835 \times(J-K)-D M}{1-17.835 / 0.66}$

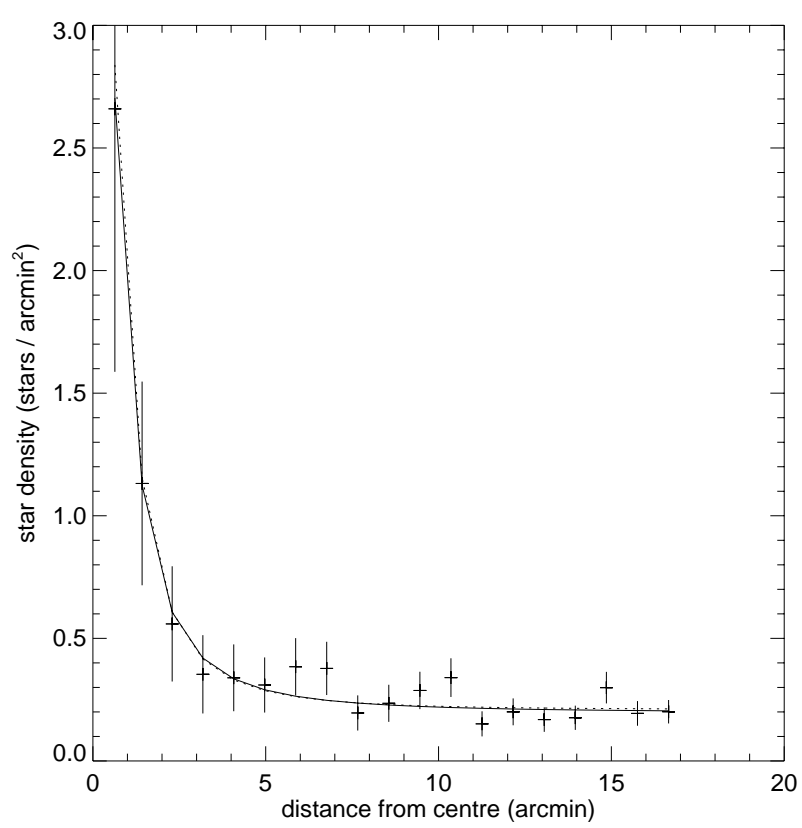

Fig. 4. Radial profile for OB stars of NGC 6910. The crosses correspond to a discretization with 19 annuli, the vertical line being proportional to the estimated error. The solid line curve is the best fitting King profile. The important point is the tail fit in order to precisely estimate the field star density.
This approximation is very precise for $\mathrm{B}$ stars. For other spectral types, it leads to a slight overestimation of $A_{K}$ (5 to $8 \%$ in the O6-F5 range). This error is negligible as compared to distance uncertainties (typically $\pm 0.6^{\mathrm{m}}$ ), so we will use Eq. (5) for the whole spectral range of interest. Using Eqs. (4) and (5), one can define a selection box which corresponds to stars respecting the two following criteria:

$\tilde{K}_{\text {low }} \geq K-D M-0.66 \times(J-K) \geq \tilde{K}_{\text {up }}$

$A_{K, \text { low }} \geq A_{K} \geq A_{K, \text { up }}$.

The first criteria allows to select the star population to be analyzed with respect to spectral types in a way independent of reddening. The second criteria was used to remove stars with an absorption very different from the typical cluster absorption. The $A_{K}$ selection is particularly efficient in improving the cluster to field star ratio, leading to a much more reliable morphology characterization and radial profile analysis.

The CMD is obtained by counting the number of stars within pixels of $0.2 \mathrm{mag}$ in $K$ and $0.1 \mathrm{mag}$ in $J-K$. A cluster CMD was defined by using only stars within a circular area of diameter $\phi$ around the center. The field CMD is estimated by taking into account only stars inside the square of size $a$ but outside the circular area. After normalization, the field CMD is subtracted from the cluster CMD resulting in a field star corrected CMD:

$C M D_{\text {cluster }}=C M D_{\text {on }}-C M D_{\text {off }} \times \frac{\pi \phi^{2}}{4 a^{2}-\pi \phi^{2}}$.

As example, Fig. 5 shows the CMD of NGC 6910. Theoretical main sequences are shown for reddenings of $A_{K}=0^{\mathrm{m}}$ to $3^{\mathrm{m}}$ in steps of $0.5^{\mathrm{m}}$ (the labels correspond to $10 \times A_{K}$ ). The segmented solid line in the lower part corresponds to the completeness limit estimated from the NGC 6910 field. For $J-K \leq J-K_{\lim }$ (1.5 ${ }^{\mathrm{m}}$ for NGC 6910$)$ the $K$ limit drives the sample completeness. In the other case, the $J$ limit becomes dominant. The parallelogram corresponds to a selection box as previously defined 


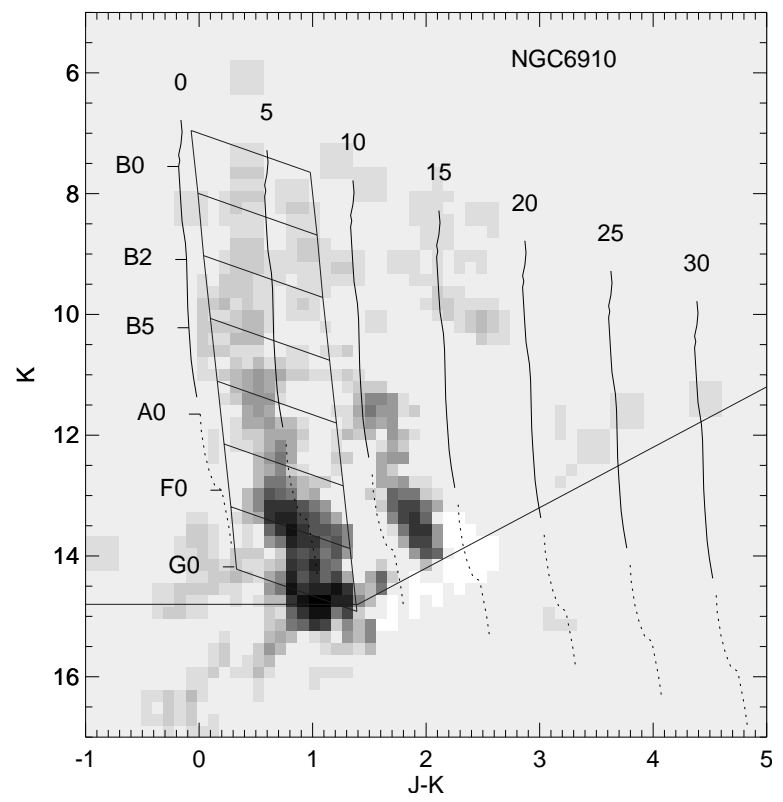

Fig. 5. Color magnitude diagram for NGC 6910 after field star subtraction The estimated completeness limit is shown as the segmented solid line. Theoretical main sequences are also shown for $A_{K}=0^{\mathrm{m}}$ to $3^{\mathrm{m}}$ in steps of $0.5^{\mathrm{m}}$ with $\mathrm{DM}=11^{\mathrm{m}}$. Labels correspond to $10 \times A_{K}$. The selection boxes correspond to O6 to G0 stars with $0.1^{\mathrm{m}}<A_{K}<0.8^{\mathrm{m}}$.

with $\mathrm{DM}=11^{\mathrm{m}},-4^{\mathrm{m}} \leq \tilde{K} \leq 3^{\mathrm{m}}, 0.1^{\mathrm{m}} \leq A_{K} \leq 0.8^{\mathrm{m}}$. The intersection of the theoretical MS corresponding to $A_{K}=A_{K \text { up }}$ with the completeness limit gives the spectral type limit for the cluster. Here, a clear main sequence appears between $A_{K}=0^{\mathrm{m}}$ and $A_{K}=0.6^{\mathrm{m}}$ confirming the presence of a very young cluster with low absorption.

\subsubsection{Mass spectrum}

To determine the mass spectrum of each cluster, we divided the CMD into 8 one magnitude wide intervals in the range $-5^{\mathrm{m}} \leq \tilde{K} \leq 3^{\mathrm{m}}$. A mean stellar mass $M_{i}$ was assigned to each interval using the the mass-luminosity relation derived in Appendix A of Paper I. The number of stars $N_{i}$ in each magnitude interval was determined by performing a radial profile analysis for the corresponding $\tilde{K}$ and $A_{K}$ intervals (see Sect. 2.2.2). The initial mass function is then approximated by $\xi\left(M_{i}\right)=\log _{10} \frac{N_{i}}{\Delta_{i}}$ where $\Delta_{i}$ is the logarithmic mass step corresponding to the respective magnitude intervals. Associated errors have been derived from the star population errors. For illustration, the IMF obtained for NGC 6910 is shown in Fig. 6.

The IMF distribution is fitted using a power law of slope $\Gamma$. Note that $\Gamma$ is theoretically independent of DM. We verified this point by repeating our analysis for various choices of DM, which, apart from mass bining effects, resulted in a constant $\Gamma$. Points affected by incompleteness, manifesting as outlying points at the low mass end, have been removed from the fit.

The luminous mass of each cluster was estimated by integrating the IMF over plausible initial mass intervals $\left[M_{\text {low }}, M_{\text {up }}\right]$. We performed the estimation with $M_{\text {up }}=$ $120 M_{\odot}$ and $M_{\text {low }}=1.0$ or $0.08 M_{\odot}$, which provides mass

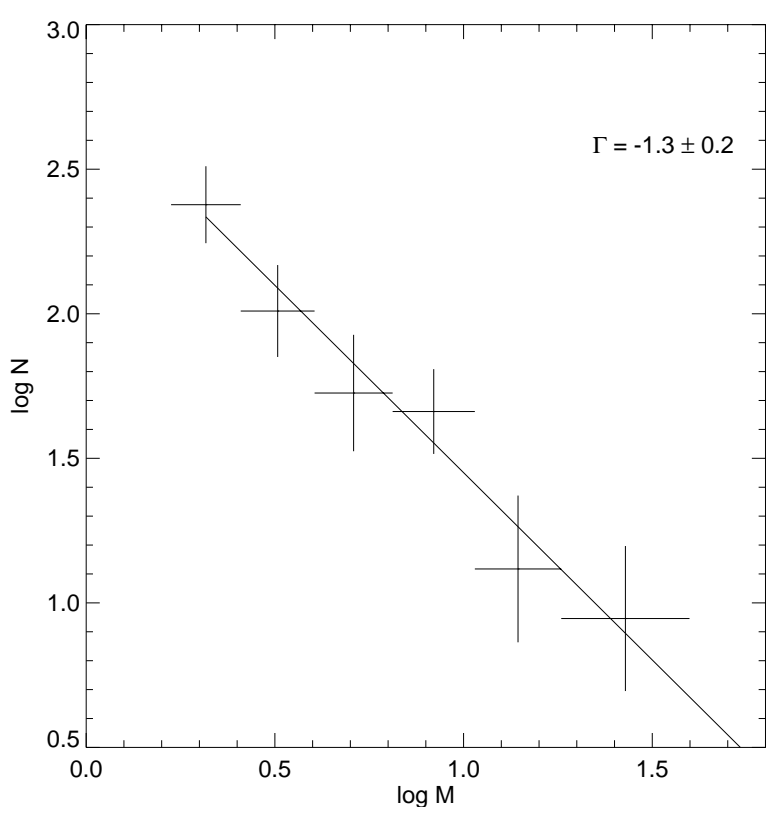

Fig. 6. IMF of NGC 6910. Only 6 mass bins are used here for the linear fit. The first bin (G0-F3) was removed because a bright foreground star hampers precise faint star counts. The last bin had no star.

estimates that bound the plausible cluster mass and reflect the uncertainties in the IMF extrapolation. In the $1-120 M_{\odot}$ range, the IMF slope is supposed to be constant and having our best fitted value. Below $1 M_{\odot}$, the IMF has to be extrapolated due to incompleteness of 2MASS data. In this work, we followed the Kroupa recommendation (Kroupa 2001) using $\Gamma_{1}=-0.3$ for $0.08 \leq M \leq 0.5 M_{\odot}$ and $\Gamma_{2}=-1.3$ for $0.5 \leq M \leq 1.0 M_{\odot}$.

\subsubsection{Iteration}

The previous steps were repeated several times for each cluster until the CMD exhibited a clear main sequence, the smoothed density maps revealed a quasi-circular morphology, the radial profiles had a smooth and decreasing shape and the $\chi^{2}$ value of the radial fit was satisfactory.

\section{Known open clusters}

For validation, we applied our method to known open clusters in the Cygnus area with sufficient population to be analyzed with our approach. These clusters are listed in Table 1 together with their distance modulus and reddening. NGC 6910 is related to the Cygnus OB9 association while NGC 6913, Berkeley 86 and IC 4996 are candidate nuclei of the Cygnus OB1 association (Garmany \& Stencel 1992). Berkeley 87 is also in the same area but slightly more distant from the central zone. These five open clusters are very young with ages varying from a few $10^{6}$ to $10^{7}$ years. Berkeley 85 and van den Bergh 130 are somewhat older and more massive. They have very few published characteristics. 
Table 1. Distance moduli and reddenings for known open clusters in the Cygnus region.

\begin{tabular}{llll}
\hline \hline Name & $\begin{array}{l}\text { DM } \\
(\mathrm{mag})\end{array}$ & $\begin{array}{l}E(B-V) \\
(\mathrm{mag})\end{array}$ & $\begin{array}{l}A_{K} \\
(\mathrm{mag})\end{array}$ \\
\hline IC 4996 & 11.5 & {$[0.5,0.8]$} & {$[0.05,0.35]$} \\
VdB 130 & 10.0 & {$[0.7,1.2]$} & {$[0.50,1.50]$} \\
Ber 85 & 11.0 & - & {$[0,0.6]$} \\
Ber 86 & 11.1 & {$[0.8,1.1]$} & {$[0.10,0.40]$} \\
Ber 87 & 11.4 & {$[1.5,1.8]$} & {$[0.35,0.65]$} \\
NGC 6910 & 11.3 & {$[0.9,1.25]$} & {$[0.20,0.50]$} \\
NGC 6913 & 11.3 & {$[0.5,1.5]$} & {$[0.10,0.50]$} \\
\hline
\end{tabular}

\subsection{Distance modulus and reddening}

When available, we used the distance moduli as deduced from our survey of young clusters based on the most recent spectroscopic data (Knödlseder et al. 2002, Paper II). For van den Bergh 130, we adopted DM $=10^{\mathrm{m}}$ (Racine 1974). For Berkeley 85, no previous distance estimate was found. We assumed that this cluster is related to the massive star associations in Cygnus for which DM $=11^{\mathrm{m}}$ presents a reasonable mean value.

The third column of Table 1 lists the $E(B-V)$ range we adopted for each cluster. For IC 4996, Ber 86, Ber 87, NGC 6910 and NGC 6913, the choice is based on the results presented in Paper II. We deduced an absorption range for the MS assuming $A_{V} \approx 10 \times A_{K}$ and $A_{V} \approx 3.1 \times E(B-V)$. We adopted a 0.1 supplementary margin to take into account uncertainties of our $A_{K}$ approximation (see Eq. (5)). These values are usually based on spectroscopy of massive stars and hence apply well to the upper part of the main sequence. The lower part often seems to extend towards more reddened stars. In this case, it is difficult to discriminate between field star contamination, young late type stars still embedded in their gas cloud, possible PMS population and a binary effect. The sensitivity of the reddening selection to the results has been explored by progressively enlarging the absorption range for $\mathrm{A}$ to $G$ stars. Note that inclusion of non-MS members in the mass spectrum determination invalidates our approach since our mass-luminosity calibration is only valid for MS members. For VdB 130, Racine (1974) reports an abnormal extinction law of $A_{V} \approx 8.1 \times E(B-V)$ with variable reddening between $E(B-V)=0.70$ to 1.2 . No data is available for Berkeley 85 and we deduced an absorption range from the CMD by enclosing the MS within a 0.1 mag margin.

\subsection{General results}

Table 2 summarizes the derived parameters for the seven known clusters. Comparison between our center coordinates and the center coordinates obtained from the SIMBAD (2002 simbad.u-strasbg. fr) and WEBDA (2002 obswww . unige . ch/webda) data bases shows a maximum discrepancy of about $0.6^{\prime}$ for the clusters with low or moderate reddening. For Ber 87 and VdB 130 the discrepancy is respectively $2.1^{\prime}$ and $4.6^{\prime}$ and results from the absorption patterns which affect differently $U B V$ and infrared surveys, modifying the apparent morphology.

The completeness is situated at spectral type F7/F8 for the four clusters with moderate absorption while it is only F3/F4 for Ber 87 and VdB 130, that are more reddened.

NGC 6910, NGC 6913, Ber 87 and VdB 130 are quite extended with comparable radii of about $8^{\prime}$. IC 4996, Ber 85 and 86 are smaller. The ratio between our $90 \%$ population radius and the radius quoted in the WEBDA database value is quite stable and varies from 1.50 to 1.63 . This probably results from the better completeness obtained with 2MASS data in these heavily reddened regions as compared to previous $U B V$ surveys.

The clusters have low or moderate population. The total OB population is estimated as $155 \pm 35$ stars. VdB 130 seems the most massive, but there is possible confusion with OB stars of the surrounding Cygnus OB1 association. In five cases, we can compare our results with the results of Paper II for stars in the 7-25 $M_{\odot}$ range. For NGC 6913 and IC 4996, results are very close. For NGC 6910 (15 \pm 4 vs. 7) it is very probable that the spectroscopic data used in Paper II is incomplete. For Ber 86 (6 \pm 3 vs. 11$)$ and Ber 87 (10 \pm 4 vs. 24$)$, the radius considered in Paper II is much greater than the value adopted in this work. It is probable that the star samples in Paper II contain non member OB stars which in fact belong to the surrounding Cyg OB1 association.

We obtain a very homogeneous IMF slope with mean value of $\Gamma=-1.30$, close to the classical Salpeter value. Except for Ber 85, we always excluded the first mass bin corresponding to F3 to G0 stars for the IMF fitting because of incompleteness and possible confusion. For IC 4996 and Ber 86, A2 to F3 stars were also removed for the same reasons. For Ber 87, only OB stars are taken into account.

For NGC 6910 and NGC 6913, our mass estimation is higher than previous estimates by Bruch (Bruch \& Sanders 1983) which are respectively 518 and $333 M_{\odot}$. For IC 4996 , the Bruch estimate is enclosed by our mass range.

\subsection{Individual results and comments}

\section{IC 4996}

IC 4996 is quite difficult to study. First, it has a rather sparse population leading to poor statistics. While the CMD indicates a rather narrow reddening range $\left(A_{K}=0.1^{\mathrm{m}}\right.$ to $\left.0.35^{\mathrm{m}}\right)$ for OB stars of the main sequence, $A_{K}$ from $0.5^{\mathrm{m}}$ to $0.8^{\mathrm{m}}$ is observed for $K \geq 12^{\mathrm{m}}$ (see Fig. 7). The morphology of this subset shows that it coincides well with the globular shape of the cluster. The corresponding stars can either be pre-main sequence stars, as suggested by Delgado \& Alfaro (1998), or unresolved binaries as suggested by Vansevicius et al. (1996). They could also simply be young, late type MS stars whose stellar winds have not yet managed to blow away the dust clouds surrounding them. The results presented in Table 2 do not include this subset and is restricted to stars on the main sequence with the absorption range stated in Table 1 . If one adds the more reddened 


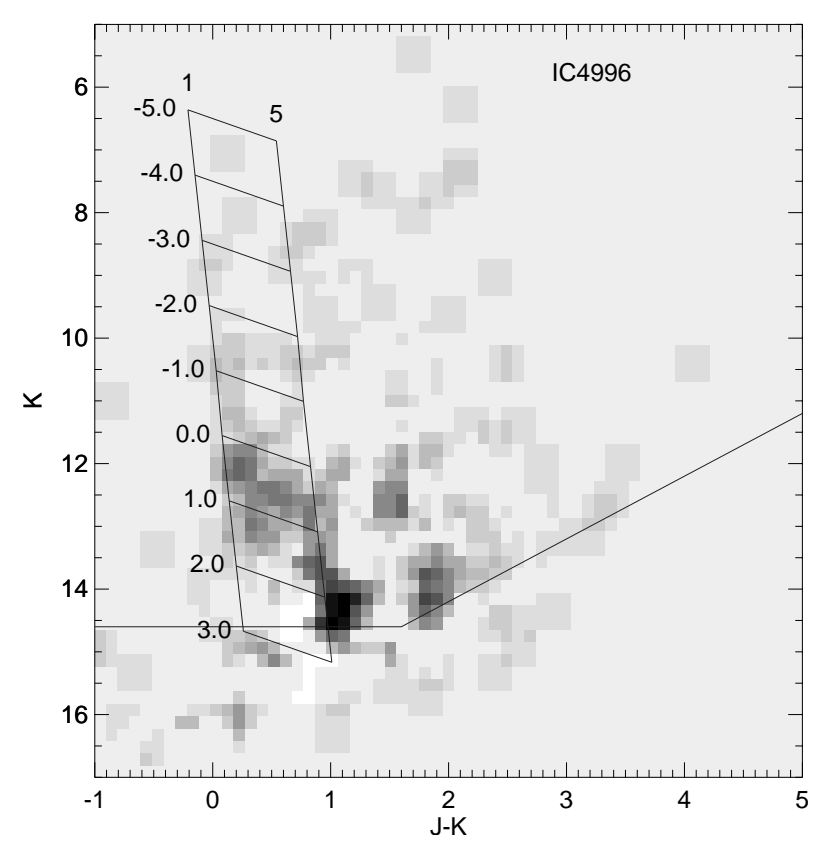

Fig. 7. IC $4996 \mathrm{CMD}$ assuming $\mathrm{DM}=11.5$. The selection boxes correspond here to $\mathrm{O} 6$ to $\mathrm{G} 0$ stars with $0.05^{\mathrm{m}}<A_{K}<0.55^{\mathrm{m}}$. In the low part, more reddened A to F stars could be PMS stars as suggested by Delgado \& Alfaro (1998).

stars, the total number of stars in the $\mathrm{O}$ to $\mathrm{F} 3$ range becomes $93 \pm 23$ and the IMF slope steepens, reaching $\Gamma=-1.6$. Yet, the $90 \%$ population radius is left unchanged, indicating that the objects are physically related to the cluster.

\section{VdB 130}

VdB 130 is affected by highly variable extinction. The morphology is clearly globular for late type stars. For more massive stars, the cluster seems to extend and is poorly defined. This situation corresponds well to a moderately old cluster with many evolved $\mathrm{O}$ and $\mathrm{B}$ stars.

\section{Ber 85}

In the CMD, a first MS star distribution with a rather low turnoff (near $K=0^{\mathrm{m}}$, B8 type assuming DM $=11^{\mathrm{m}}$ ) is detected between $A_{K}=0.2^{\mathrm{m}}$ and $A_{K}=0.5^{\mathrm{m}}$. The corresponding stars have a clear globular shape. Other MS-like star distributions appear in the CMD near $A_{K}=1.0^{\mathrm{m}}$ and $A_{K}=1.5^{\mathrm{m}}$. The corresponding stars lie to the southeast of the first structure and do not have a clear morphology. They probably correspond to superposition effects and were excluded from the analysis. The distance modulus is unknown and the results are speculative. Under the assumption of $\mathrm{DM}=11^{\mathrm{m}}$, we find only $12 \pm 8 \mathrm{OB}$ stars and $96 \pm 27 \mathrm{O}$ to $\mathrm{F} 3$ stars. The star population is then mainly of the A and F spectral type and the cluster is probably quite old. Assuming $\mathrm{DM}=10^{\mathrm{m}}$, the number of OB stars drops to $N_{\mathrm{OB}}=2$, the total number of stars in the $\mathrm{O}$ to $\mathrm{F} 3$ range to $N_{\mathrm{OF} 3}=23$ and the mass of $\mathrm{O}$ to $\mathrm{GO}$ stars is $M_{\text {low }}=380 M_{\odot}$. With $\mathrm{DM}=12^{\mathrm{m}}$, we have $N_{\mathrm{OB}}=61$ and $M_{\text {low }}=1100 M_{\odot}$. The IMF slope is independent of DM except for mass bining effects: $\Gamma=-1.47 \pm 0.2$.

\section{Ber 86}

The case of Ber 86 is quite similar to that of IC 4996. The MS tends to widen in the lower part and $F$ and $G$ stars seem more reddened than the selected reddening range $\left(A_{K}\right.$ can reach $0.7^{\mathrm{m}}$ ). This population coincides well with the cluster morphology. If this population is excluded from the analysis, almost no A to $\mathrm{G}$ stars are found. Taking it into account leads to $67 \pm 19$ stars from $\mathrm{O}$ to $\mathrm{F} 3$ type. In this case, the IMF slope becomes $-1.34 \pm 0.29$. Massey \& Johnson (1995) estimate a total of 10 stars with mass greater than $10 M_{\odot}$ to be compared to our $14 \pm 6$ estimation of stars having mass greater than $3.4 M_{\odot}$. A recent study based on CCD photometry (Deeg \& Ninkov 1996) found 16 stars in the same range. In this work, the estimated IMF slope is $-1.3 \pm 0.3$ without inclusion of PMS stars or binaries and $-1.4 \pm 0.4$ with this population, in good accordance with our results. Our IMF exhibits a gap for B2 to B7 stars (4.5 to $10 M_{\odot}$ ), more pronounced towards early B stars, similar to the results found in the Deeg \& Ninkov (1996) study.

\section{Ber 87}

Two bright foreground stars and heavy absorption severely hamper the Ber 87 study. A2 to G0 samples are considerably incomplete. Only OB stars have good completeness. They seem to extend to a rather large radius of about $8^{\prime}$, a value also found by Turner $\&$ Forbes (1982). Their study on Ber 87 was based on UBV photometry with a magnitude limit of $17^{\mathrm{m}}$. Assuming $\mathrm{DM}=11.4^{\mathrm{m}}$ and $A_{V \max }=5.7^{\mathrm{m}}$, their sample was complete only for O to B8 stars. They estimated a total of 40 stars and concluded that this cluster was more massive than previously thought and only apparently sparsely populated because of the heavy absorption in the UBV range. We agree with this interpretation although our result $(26 \pm 8$ OB stars) moderates it. The OB star population and the overall size make Ber 87 comparable to NGC 6910.

\section{NGC 6910}

NGC 6910 has a clear main sequence distribution centered on $A_{K}=0.35^{\mathrm{m}}$. For F3 to G0 stars, the MS widens slightly up to $A_{K}=0.6^{\mathrm{m}}$. A second main sequence-like structure appears near $A_{K}=1.1^{\mathrm{m}}$ (see Fig. 5). Density maps show that the corresponding stars belong to another structure on the edge of the field, probably unrelated to NGC 6910. The effects of two bright foreground stars on photometry are efficiently compensated by our masking procedure (see Fig. 2) and the obtained cluster morphology is clear (see Fig. 3), except for the fainter stars where field star contamination is difficult to avoid. As can be seen in Fig. 6, the linear fit of the IMF is good and led to a slope close to the Salpeter value. Performing the analysis with $A_{K \text { up }}=0.7^{\mathrm{m}}$ for A to $\mathrm{G}$ stars, leads to $132 \pm 30 \mathrm{O}$ to $\mathrm{F} 3$ stars and the IMF slope becomes $-1.36 \pm 0.22$.

\section{NGC 6913}

NGC 6913 exhibits a clear MS distribution centered on $A_{K}=$ $0.40^{\mathrm{m}}$. Star counts are in good agreement with the result 
Table 2. Characteristics of known open clusters in the Cygnus area.

\begin{tabular}{|c|c|c|c|c|c|c|c|c|c|c|}
\hline Name & $\begin{array}{l}\alpha^{a} \\
(\mathrm{hms})\end{array}$ & $\begin{array}{l}\delta^{a} \\
(\mathrm{dms})\end{array}$ & $\begin{array}{l}\tilde{K}_{\lim }{ }^{b} \\
\text { (type) }\end{array}$ & $\begin{array}{l}R_{90}{ }^{c} \\
\left(^{\prime}\right)\end{array}$ & $\begin{array}{l}N_{\mathrm{OF} 3}^{d} \\
\text { (stars) }\end{array}$ & $\begin{array}{l}N_{\mathrm{OB}}^{e} \\
\text { (stars) }\end{array}$ & $\begin{array}{l}\rho_{c}{ }^{f} \\
\left(* / \operatorname{arcmin}^{2}\right)\end{array}$ & $-\Gamma^{g}$ & $\begin{array}{l}M_{\text {low }}^{h} \\
\left(M_{\odot}\right)\end{array}$ & $\begin{array}{l}M_{\text {up }}{ }^{h} \\
\left(M_{\odot}\right)\end{array}$ \\
\hline IC 4996 & $20^{\mathrm{h}} 16^{\mathrm{m}} 30^{\mathrm{s}}$ & $+37^{\circ} 37^{\prime} 59^{\prime \prime}$ & $\mathrm{F} 4$ & 3.4 & $47 \pm 16^{*}$ & $10 \pm 6$ & $2.7 \pm 1.0$ & $1.43 \pm 0.30$ & 350 & 890 \\
\hline VdB 130 & $20^{\mathrm{h}} 18^{\mathrm{m}} 00^{\mathrm{s}}$ & $+39^{\circ} 19^{\prime} 48^{\prime \prime}$ & $\mathrm{F} 3$ & 8.7 & $103 \pm 51$ & $46 \pm 28$ & $1.0 \pm 0.5$ & $1.28 \pm 0.25$ & 650 & 1460 \\
\hline Ber 85 & $20^{\mathrm{h}} 18^{\mathrm{m}} 51^{\mathrm{s}}$ & $+37^{\circ} 43^{\prime} 01^{\prime \prime}$ & G0 & 5.5 & $96 \pm 27$ & $12 \pm 8$ & $0.6 \pm 0.3$ & $1.46 \pm 0.18$ & 780 & 1940 \\
\hline Ber 86 & $20^{\mathrm{h}} 20^{\mathrm{m}} 12^{\mathrm{s}}$ & $+38^{\circ} 41^{\prime} 24^{\prime \prime}$ & F7 & 4.6 & $14 \pm 8^{*}$ & $14 \pm 6$ & $0.6 \pm 0.3$ & $1.23 \pm 0.31$ & 330 & 770 \\
\hline Ber 87 & $20^{\mathrm{h}} 21^{\mathrm{m}} 34^{\mathrm{s}}$ & $+37^{\circ} 23^{\prime} 06^{\prime \prime}$ & F7 & 8.1 & $39 \pm 21^{* *}$ & $26 \pm 8$ & $1.8 \pm 0.6$ & $1.35 \pm 0.40$ & 510 & 1380 \\
\hline NGC 6910 & $20^{\mathrm{h}} 23^{\mathrm{m}} 08^{\mathrm{s}}$ & $+40^{\circ} 46^{\prime} 30^{\prime \prime}$ & F7 & 8.3 & $106 \pm 27$ & $30 \pm 10$ & $2.6 \pm 1.1$ & $1.30 \pm 0.21$ & 580 & 1300 \\
\hline NGC 6913 & $20^{\mathrm{h}} 23^{\mathrm{m}} 56^{\mathrm{s}}$ & $+38^{\circ} 31^{\prime} 23^{\prime \prime}$ & F8 & 7.3 & $78 \pm 23$ & $17 \pm 10$ & $0.9 \pm 0.4$ & $1.09 \pm 0.17$ & 580 & 1090 \\
\hline
\end{tabular}

Table 3. Characteristics of new cluster candidates in the Cygnus area.

\begin{tabular}{|c|c|c|c|c|c|c|c|c|c|c|c|}
\hline Name & $\begin{array}{l}\alpha^{a} \\
(\mathrm{hms})\end{array}$ & $\begin{array}{l}\delta^{a} \\
(\mathrm{dms})\end{array}$ & $\begin{array}{l}\tilde{K}_{\text {lim }}{ }^{b} \\
\text { (type) }\end{array}$ & $\begin{array}{l}R_{90}{ }^{c} \\
\left(^{\prime}\right)\end{array}$ & $\begin{array}{l}N_{\mathrm{OB}}^{e} \\
\text { (stars) }\end{array}$ & $\begin{array}{l}\rho_{\mathrm{c}}{ }^{f} \\
\left(* / \operatorname{arcmin}^{2}\right)\end{array}$ & $-\Gamma^{g}$ & $\begin{array}{l}M_{\text {low }}^{h} \\
\left(M_{\odot}\right)\end{array}$ & $\begin{array}{l}M_{\text {up }}{ }^{h} \\
\left(M_{\odot}\right)\end{array}$ & $\mathrm{HII}^{i}$ & Ref. $^{j}$ \\
\hline $\mathrm{Cl} 01$ & $20^{\mathrm{h}} 24^{\mathrm{m}} 25^{\mathrm{s}}$ & $+36^{\circ} 24^{\prime} 18^{\prime \prime}$ & $\mathrm{A} 4$ & 3.0 & $35 \pm 10$ & $8.6 \pm 2.5$ & $1.48 \pm 0.28$ & 1100 & 3130 & - & - \\
\hline $\mathrm{ClO} 2$ & $20^{\mathrm{h}} 27^{\mathrm{m}} 25^{\mathrm{s}}$ & $+37^{\circ} 22^{\prime} 48^{\prime \prime}$ & A1 & 1.1 & $20 \pm 5$ & $14.0 \pm 5.0$ & $0.93 \pm 0.37$ & 400 & 600 & S106 & - \\
\hline $\mathrm{ClO} 3$ & $20^{\mathrm{h}} 25^{\mathrm{m}} 38^{\mathrm{s}}$ & $+39^{\circ} 24^{\prime} 36^{\prime \prime}$ & A5 & 4.9 & $31 \pm 10$ & $4.0 \pm 1.5$ & $1.45 \pm 0.23$ & 640 & 1330 & - & - \\
\hline $\mathrm{Cl} 04$ & $20^{\mathrm{h}} 29^{\mathrm{m}} 36^{\mathrm{s}}$ & $+39^{\circ} 01^{\prime} 48^{\prime \prime}$ & B5 & 1.2 & $15 \pm 7$ & $6.2 \pm 2$ & $1.04 \pm 0.60$ & 530 & 920 & DR9 & 6 \\
\hline $\mathrm{Cl} 05$ & $20^{\mathrm{h}} 27^{\mathrm{m}} 12^{\mathrm{s}}$ & $+39^{\circ} 26^{\prime} 24^{\prime \prime}$ & B5 & 1.8 & $34 \pm 8$ & $13.0 \pm 3$ & $1.48 \pm 0.25$ & 520 & 1370 & DR6 & 7 \\
\hline $\mathrm{Cl} 06$ & $20^{\mathrm{h}} 31^{\mathrm{m}} 45^{\mathrm{s}}$ & $+38^{\circ} 58^{\prime} 01^{\prime \prime}$ & B5 & 4.8 & $32 \pm 17$ & $10.0 \pm 2.5$ & $1.17 \pm 0.35$ & 1120 & 2210 & DR13 & 8 \\
\hline $\mathrm{Cl} 07$ & $20^{\mathrm{h}} 32^{\mathrm{m}} 28^{\mathrm{s}}$ & $+38^{\circ} 51^{\prime} 25^{\prime \prime}$ & B3 & 2.3 & $21 \pm 9$ & $8.6 \pm 2.5$ & $0.97 \pm 0.40$ & 520 & 920 & IRAS20306 & 9 \\
\hline $\mathrm{Cl} 08$ & $20^{\mathrm{h}} 32^{\mathrm{m}} 29^{\mathrm{s}}$ & $+40^{\circ} 16^{\prime} 30^{\prime \prime}$ & B5 & 4.4 & $22 \pm 9$ & $5.6 \pm 2$ & $1.39 \pm 0.20$ & 510 & 1550 & DR15 & 10 \\
\hline $\mathrm{Cl} 09^{k}$ & $20^{\mathrm{h}} 28^{\mathrm{m}} 12^{\mathrm{s}}$ & $+40^{\circ} 51^{\prime} 54^{\prime \prime}$ & B8 & 2.0 & $21 \pm 7$ & $5.8 \pm 1.5$ & $1.48 \pm 0.25$ & 440 & 1080 & DR7 & 11 \\
\hline $\mathrm{Cl} 10$ & $20^{\mathrm{h}} 39^{\mathrm{m}} 13^{\mathrm{s}}$ & $+39^{\circ} 58^{\prime} 37^{\prime \prime}$ & F5 & 6.7 & $23 \pm 9$ & $1.0 \pm 0.3$ & $1.98 \pm 0.25$ & 1010 & 4100 & - & - \\
\hline $\mathrm{Cl} 11$ & $20^{\mathrm{h}} 39^{\mathrm{m}} 34^{\mathrm{s}}$ & $+41^{\circ} 18^{\prime} 36^{\prime \prime}$ & B9 & 4.8 & $77 \pm 18$ & $11.0 \pm 3$ & $1.82 \pm 0.25$ & 2070 & 8000 & DR 22 & 13 \\
\hline $\mathrm{Cl} 12$ & $20^{\mathrm{h}} 35^{\mathrm{m}} 22^{\mathrm{s}}$ & $+42^{\circ} 21^{\prime} 36^{\prime \prime}$ & $\mathrm{A} 0$ & 2.6 & $37 \pm 8$ & $7.0 \pm 2.5$ & $1.61 \pm 0.25$ & 600 & 1760 & DR17 & 15 \\
\hline $\mathrm{Cl} 13$ & $20^{\mathrm{h}} 38^{\mathrm{m}} 29^{\mathrm{s}}$ & $+42^{\circ} 06^{\prime} 25^{\prime \prime}$ & B5 & 1.8 & $12 \pm 8^{* *}$ & $9.0 \pm 2.5$ & $1.34 \pm 0.30$ & 280 & 630 & {$[\mathrm{~L} 89 \mathrm{~b}] 81.480$} & 16 \\
\hline $\mathrm{Cl} 14$ & $20^{\mathrm{h}} 35^{\mathrm{m}} 43^{\mathrm{s}}$ & $+42^{\circ} 29^{\prime} 24^{\prime \prime}$ & F3 & 5.3 & $12 \pm 4$ & $4.6 \pm 1.5$ & $1.51 \pm 0.35$ & 470 & 1160 & - & 13 \\
\hline $\mathrm{Cl} 15$ & $20^{\mathrm{h}} 40^{\mathrm{m}} 29^{\mathrm{s}}$ & $+42^{\circ} 01^{\prime} 48^{\prime \prime}$ & $\mathrm{A} 0$ & 6.9 & $62 \pm 20$ & $4.2 \pm 1.5$ & $1.49 \pm 0.25$ & 720 & 1530 & DR23 & 17 \\
\hline
\end{tabular}

${ }^{a}$ J2000, optimized to reduce the radial extent of the O to F3 population.

${ }^{b}$ Spectral type corresponding to the estimated completeness limit of the cluster.

$c 90 \%$ population radius estimated from cumulative star counts versus radius.

${ }^{d}$ Total number of $\mathrm{O}$ to $\mathrm{F} 3$ stars in the cluster.

${ }^{e}$ Total number of $\mathrm{O}$ to $\mathrm{B}$ stars in the cluster.

${ }^{f}$ Central OB stars density.

${ }^{g}$ IMF slope, incomplete lower mass bin removed from the linear fit.

${ }^{h}$ Integration of the IMF using a constant slope above $1 M_{\odot}$ and the Kroupa description below.

$M_{\text {low }}$ corresponds to a lower cut off at $0.08 M_{\odot}$

$M_{\text {up }}$ corresponds to a lower cut off at $1.0 M_{\odot}$.

${ }^{i}$ Identified coincident HII region (see text for more details); [L89b] refers to the radio survey by Lockman (1989).

${ }^{j}$ Cluster number in Dutra \& Bica (2001).

${ }^{k}$ Assuming $\mathrm{Cl} 09$ belongs to the local arm $(\mathrm{DM}=11)$. See text for results with $\mathrm{Cl} 09$ located in the Perseus arm $(\mathrm{DM}=14)$.

* Does not include a more reddened F and G population (see text).

** Affected by severe incompleteness caused by bright foreground stars.

of 14 OB stars from Crawford \& Barnes (1977). A more recent spectroscopic study (Wang \& Hu 2000) counts 42 OB stars. Half of them are of giant class and the proportion of true members is estimated to be $70 \%$, which leaves $15 \mathrm{MS}$ stars, in good agreement with our results.

\section{New cluster candidates}

Most of the new cluster candidates are found in heavily obscured regions in the Cyg OB1, OB2 and OB9 area where clusters could not be identified using $U B V$ surveys. 


\subsection{Distance modulus and reddening}

We started our analysis by assuming a distance modulus of $11^{\mathrm{m}}$ for all clusters candidates. This corresponds to a reasonable value for the massive star associations in this area (Knödlseder et al. 2002). Note that the DM only impacts the total cluster mass determination, the total number of OB stars and the completeness limit estimation. All other parameters in Table 3 are unaffected by the choice of DM. We then refined the DM estimates by using distance estimates of probable counterparts when available. The absorption ranges were derived from the CMD analysis combined with the morphological analysis in an iterative process. The adopted values are summarized in Table 4.

\subsection{General results}

Table 3 lists the cluster candidates characteristics we derived from the 2MASS data assuming the parameters of Table 4. The columns are identical to those of Table 2 except that we discarded the OF3 population estimates because of incompleteness.

The completeness limit estimation is directly correlated to the reddening. 7 clusters are very heavily obscured with $A_{K}$ reaching 3 or even 4 . They are only complete for the $\mathrm{O}$ to $\mathrm{B} 3 / \mathrm{B} 5$ range. For these clusters, even the $\mathrm{OB}$ population is underestimated. When $A_{K}<2^{\mathrm{m}}$ or DM $<10^{\mathrm{m}}$, completeness is achieved for OB stars ( 8 cases). Only two clusters have moderate reddening and their completeness limit is situated at spectral type F3/F5.

The total estimated OB population in the new cluster candidates is $454 \pm 90$. The uncertainty reflects the uncertainty on the distance estimates and the star counts errors. $\mathrm{Cl} 11$ and $\mathrm{Cl} 15$ are the most important contributors. Yet $\mathrm{Cl} 05, \mathrm{Cl} 06$, $\mathrm{Cl} 07, \mathrm{Cl} 08$ and $\mathrm{Cl} 09$ may have a more important OB population than quoted in Table 3 because of the incompleteness affecting B stars due to extreme obscuration. We estimate that the associated underestimation of the total OB population may be around 50 stars.

Most of the new cluster candidates have a central density much higher than the central density of known clusters. This point confirms that they are very compact structures, probably very young or even nascent.

Due to the relatively low completeness magnitudes, the IMF slope of the new cluster candidates is less precisely estimated and more scattered than for the well known clusters. Nevertheless an interesting point is that the mean value is -1.40 , close to the classical Salpeter value and coherent with the mean value for the known clusters.

For most of the studied clusters, the 2MASS PSC contains one or more bright stars very close to our derived center and with reddening compatible with the mean cluster reddening. Table 5 gives the brightest star within $25 \%$ of the cluster radius. The spectral type is based on the assumption of a main sequence luminosity class $\mathrm{V}$ star and the distance modulus estimate quoted in Table 4. Compatible reddening and immediate vicinity to the center give a good membership probability but
Table 4. Estimated distance moduli and reddenings for new cluster candidates.

\begin{tabular}{llllll}
\hline \hline Name & $\begin{array}{l}\mathrm{DM} \\
(\mathrm{mag})\end{array}$ & $\begin{array}{l}A_{K} \\
(\mathrm{mag})\end{array}$ & Name & $\begin{array}{l}\mathrm{DM} \\
(\mathrm{mag})\end{array}$ & $\begin{array}{l}A_{K} \\
(\mathrm{mag})\end{array}$ \\
\hline $\mathrm{Cl} 01$ & 11.0 & {$[1.0,2.0]$} & $\mathrm{Cl} 09$ & 11.0 & {$[1.0,2.5]$} \\
$\mathrm{Cl} 02$ & 9.0 & {$[0.5,4.0]$} & $\mathrm{Cl} 10$ & 11.0 & {$[0.6,1.2]$} \\
$\mathrm{Cl} 03$ & 11.0 & {$[1.0,2.0]$} & $\mathrm{Cl} 11$ & 11.0 & {$[1.0,2.5]$} \\
$\mathrm{Cl} 04$ & 11.0 & {$[1.0,3.0]$} & $\mathrm{Cl} 12$ & 11.0 & {$[0.9,2.0]$} \\
$\mathrm{Cl} 05$ & 11.0 & {$[1.0,3.0]$} & $\mathrm{Cl} 13$ & 11.0 & {$[1.0,3.0]$} \\
$\mathrm{Cl} 06$ & 11.0 & {$[1.0,3.0]$} & $\mathrm{Cl} 14$ & 10.5 & {$[0.5,1.5]$} \\
$\mathrm{Cl} 07$ & 11.0 & {$[1.5,3.5]$} & $\mathrm{Cl} 15$ & 11.0 & {$[1.0,2.5]$} \\
$\mathrm{Cl} 08$ & 10.0 & {$[1.5,3.5]$} & & & \\
\hline
\end{tabular}

no firm evidence. Some of these stars may not be true cluster members.

For comparison, the last column of Table 5 gives an estimation of the spectral type derived from the IR luminosity of the coincident HII region. The total IR luminosity comes from various references (see the footnote of Table 5). The distance modulus is taken from Table 4 . The estimation assumes that the HII region is powered by its most luminous star and that the radiation from the stellar photosphere is totally absorbed and reradiated in the IR by the circumstellar dust. The differences between the two last columns are discussed later.

Due to extreme confusion, we could not characterize an apparent star concentration with the following approximate coordinates $\alpha=20^{\mathrm{h}} 30^{\mathrm{m}} 28^{\mathrm{s}}, \delta=+40^{\circ} 15^{\prime} 47^{\prime \prime}$. It coincides with IRAS $20286+4105$. Note also that we did not detect in the 2 MASS survey any star concentration possibly associated with DR19, DR20 and DR21.

\subsection{Individual results and comments}

\section{$\mathrm{Cl} 01$}

We have not identified any possible counterpart for this cluster. The closest radio or IR sources are more than $2.5^{\prime}$ away. We adopted here our DM default value of $11^{\mathrm{m}}$. The morphology reveals a very regular globular shape with a $3.0^{\prime}$ radius. The cluster has a clear MS distribution centered on $A_{K}=1.5^{\mathrm{m}}$. The turn-off point is near the spectral type B3 and there is no O star. At $\mathrm{DM}=11^{\mathrm{m}}$ we have $N_{\mathrm{OB}}=31 \pm 10$ and $M_{\text {low }}=$ $1100 M_{\odot} . \mathrm{DM}=10^{\mathrm{m}}$ would lead to $N_{\mathrm{OB}}=6 \pm 2$ and $M_{\text {low }}=$ $640 M_{\odot}$ while $\mathrm{DM}=12^{\mathrm{m}}$ would lead to $N_{\mathrm{OB}}=80 \pm 23$ and $M_{\text {low }}=4760 M_{\odot}$. The absence of a coincident HII region and the rather low turn-off suggest that we have here probably a rather evolved cluster.

\section{$\mathrm{Cl} 02$}

This object is associated to the bipolar S106 HII region which is powered by its central star S106 IRS4. Near infrared spectra, colors and a total luminosity of about $10^{4} L_{\odot}$ (derived from the IR luminosity of the coincident HII region) indicate that it is of late O or early B type (Bally et al. 1998), assuming a distance estimate of $\mathrm{DM}=9^{\mathrm{m}}$ (Staude et al. 1982). The cluster is surrounded by a torus of molecular gas, already broken up in 
individual clumps (Loushin et al. 1990; Barsony et al. 1989). The morphology reveals here a very dense and small globular structure surrounded by a ring that is almost devoid of stars and that corresponds well to the torus cited above. Our 1.1' radius may only correspond to the core of the structure. The associated stars have a very scattered CMD with extremely variable and heavy absorption, ranging from $A_{K}=1.0^{\mathrm{m}}$ to $A_{K}=4.0^{\mathrm{m}}$. The stellar content is difficult to characterize and 2MASS data may be unreliable in this very crowded area with possibly many pre-main sequence stars. Only OB stars are a reliable sample in this context. They appear very concentrated near the center where the star density peaks at a high value of $14 \mathrm{stars} / \mathrm{arcmin}^{2}$. The IMF calculated from these massive stars is unusually flat. It is possible that due to the extreme young age of the structure, even massive stars are still in the PMS stage and our calibration is no longer valid. The 2MASS PSC contains a very bright and reddened star $27^{\prime \prime}$ away from the cluster center. Its coordinates correspond exactly to IRS4. We derive $A_{K}=2.8^{\mathrm{m}}$ from Eq. (5). Assuming this value, we obtain $M_{K}=-5.8^{\mathrm{m}}$. With the absorption derived by Bally et al. (1998), $A_{K}=2.2^{\mathrm{m}}$, we have $M_{K}=-5 \cdot 3^{\mathrm{m}}$. Both results are incompatible with a single MS star at DM $=9$ and also differ substantially from the magnitude derived from the IR luminosity of the coincident HII region (see Table 5). This discrepancy implies that either the 2MASS photometry is invalid because of the crowded nature of the area and the presence of bright nebulosity or that the total luminosity derived from far IR flux is underestimated. In this second case we may have either a binary star, as suggested by the two radio peaks observed by Hoare et al. (1996) or a supergiant class star, which is however not compatible with the supposed extreme young age of the cluster or a shorter distance.

\section{$\mathrm{Cl} 03$}

The most nearby counterpart to this cluster is IRAS $20237+3915$ at $64^{\prime \prime}$ from the center. The other objects we found are two radio sources and a nebula, but they lie more than $5^{\prime}$ away from the cluster center. IRAS $20237+3915$ has $S_{12 \mu \mathrm{m}}=0.8 \mathrm{Jy}, S_{25 \mu \mathrm{m}}=1.5 \mathrm{Jy}, S_{60 \mu \mathrm{m}}=35.7 \mathrm{Jy}$, $S_{100 \mu \mathrm{m}}=172.2 \mathrm{Jy}$. According to the criterion presented by Taylor et al. (1996), it is not a compact HII region. Using the flux estimation provided by Casoli et al. (1986), we derive $L=370 L_{\odot}$ at $\mathrm{DM}=11^{\mathrm{m}}$ which corresponds to a B7 star. The cluster has a globular shape of $5^{\prime}$ radius surrounded by a ring of very obscured regions. The central part has a moderate star concentration. The CMD shows a clear MS distribution with $1.0^{\mathrm{m}} \leq A_{K} \leq 2.0^{\mathrm{m}}$. The turn-off point is near spectral type B3. Assuming DM $=11^{\mathrm{m}}, \mathrm{Cl} 03$ is rather massive with about $30 \mathrm{OB}$ stars. $N_{\mathrm{OB}}$ varies from 16 at $\mathrm{DM}=10^{\mathrm{m}}$ to 80 at $\mathrm{DM}=12^{\mathrm{m}}$. From the PSC, we found a bright star 15" away from the cluster center with compatible reddening. Assuming $\mathrm{DM}=11^{\mathrm{m}}$, we would have $M_{K}=-4.6^{\mathrm{m}}$, corresponding to a O4V star. At DM $=10^{\mathrm{m}}$, we would have a $08.5 \mathrm{~V}$ star. The presence of such a bright MS star without any important HII region is not probable. Although it is possible that strong stellar winds have created a cavity that prevents the formation of a compact HII region, it is more probable that the above star is either an evolved giant, which possibly is more compatible with the estimated MS turn-off type of B3, or a foreground star that lies closer. $\mathrm{Cl} 03$ is probably an evolved cluster in the local arm, although the possibility of a very massive and more distant object is not completely ruled out.

\section{$\mathrm{Cl} 04$}

This object coincides with a concentration of radio sources and the HII region DR9 (40" from the center). IRAS $20277+3851$ is also very close. Distance estimates for DR9 from the literature are very scattered, ranging from DM = $10.3^{\mathrm{m}}$ (Odenwald 1989) to $\mathrm{DM}=13.3^{\mathrm{m}}$ (Kurtz et al. 1994). The most recent infrared study (Comerón \& Torra 2001) indicates that the short hypothesis is to be preferred. We will assume a default value DM $=11^{\mathrm{m}}$ which is also employed in Dutra's work (Dutra \& Bica 2001). The CMD shows two groups of stars corresponding to the same globular and very compact object (1.1' radius). The first is situated around $A_{K}=$ $1.5^{\mathrm{m}}$ and contains only a few bright stars while the second has $A_{K}=2.4^{\mathrm{m}}$ showing a clear MS distribution. This may indicate that we have a highly variable extinction across the cluster region. A chance superposition seems unlikely due to the compactness of the structure. The radius may only be apparently small and corresponds probably only to the core. The situation is quite confused and the results are unreliable because of poor completeness. From the 2MASS PSC, we find a bright star $16^{\prime \prime}$ away from the cluster center with compatible reddening. Assuming $\mathrm{DM}=11^{\mathrm{m}}$, we have $M_{K}=-4.6^{\mathrm{m}}$ consistent with an $\mathrm{O} 4 \mathrm{~V}$ main sequence star or a giant B star. $\mathrm{DM}=10^{\mathrm{m}}$ would lead to a O7V star and would reduce the total number of OB star to $7 \pm 3$. DM $=13^{\mathrm{m}}$ would lead to an extremely luminous supergiant and to a total of about $60 \mathrm{OB}$ stars. The preceding results are consistent with the picture of a very compact young cluster with a central powering late $\mathrm{O}$ star having DM between $10^{\mathrm{m}}$ and $11^{\mathrm{m}}$, analogous to $\mathrm{Cl} 02$. Yet, they do not completely rule out the possibility of a more massive and distant cluster.

\section{$\mathrm{Cl} 05$}

This object is clearly associated with the HII region DR6 (41" from the center). It is very analogous to $\mathrm{Cl} 02$ and $\mathrm{Cl} 04$, with a slightly greater radius of $1.8^{\prime}$. DR6 distance estimates vary between $\mathrm{DM}=10^{\mathrm{m}}$ (Comerón \& Torra 2001), $\mathrm{DM}=10.4^{\mathrm{m}}$ (Dutra \& Bica 2001) and DM $=10.9^{\mathrm{m}}$ (Odenwald et al. 1986). The CMD also features two groups of stars with similar morphology yet different obscuration. The first is situated around $A_{K}=1.5^{\mathrm{m}}$ and the second has $A_{K}=$ $2.4^{\mathrm{m}}$ with a clear MS distribution. From the PSC, we find two bright stars $21^{\prime \prime}$ and $44^{\prime \prime}$ away from the cluster center and with compatible reddening. Assuming DM $=11^{\mathrm{m}}$, we would have $M_{K}=-3.88^{\mathrm{m}}$ and $-4.06^{\mathrm{m}}$ consistent with $07 \mathrm{~V} / 07.5 \mathrm{~V}$ main sequence stars. $\mathrm{DM}=10^{\mathrm{m}}$ would lead to $N_{\mathrm{OB}}=13 \pm 3$ and $M_{\text {low }}=280 M_{\odot}$ while DM $=12^{\mathrm{m}}$ would lead to $N_{\mathrm{OB}}=56 \pm 13$ and $M_{\text {low }}=1100 M_{\odot}$. Our results fit well into the picture of a compact young cluster powered by late $\mathrm{O}$ stars located between $\mathrm{DM}=10^{\mathrm{m}}$ and $\mathrm{DM}=11^{\mathrm{m}}$. 
This object coincides with a concentration of radio sources and the HII region [L89b]78.163-0.367 (37" from the center). The center of the HII region DR13 is located 4' away from the cluster center. Dutra \& Bica (2001) estimate the distance of this cluster to be $\mathrm{DM}=10.9^{\mathrm{m}}$. According to Odenwald \& Schwarz (1993), DR 13 lies at $1.2-1.5 \mathrm{kpc}$. The CMD is very scattered and the absorption varies from $A_{K}=1.0^{\mathrm{m}}$ to $A_{K}=3.0^{\mathrm{m}}$. The morphology is very clear, with an apparently denser core. Radial profiles are very regular with a high central star density of $10 \pm 2.5 \mathrm{stars} / \mathrm{arcmin}^{2}$. From the PSC, we find a bright star $2.5^{\prime \prime}$ away from the cluster center and with compatible reddening. Assuming DM $=11^{\mathrm{m}}$, we have $M_{K}=-3.8^{\mathrm{m}}$ consistent with an $07.5 \mathrm{~V}$ main sequence star. $\mathrm{DM}=10^{\mathrm{m}}$ would lead to a B0.5V star, $N_{\mathrm{OB}}=12 \pm 6$ and $M_{\text {low }}=510 M_{\odot}$ while $\mathrm{DM}=12^{\mathrm{m}}$ would lead to an O3V star, $N_{\mathrm{OB}}=105 \pm 55$ and $M_{\text {low }}=1575 M_{\odot}$. This object is probably of the same class as $\mathrm{Cl} 02, \mathrm{ClO} 4$ and $\mathrm{Cl} 05$, but larger and possibly powered by a single central late $\mathrm{O}$ star.

\section{$\mathrm{Cl} 07$}

This is a small and very obscured cluster. It lies $14.1^{\prime}$ to the south east of the center of DR13. It is associated to IRAS 20306+3841 ( $6^{\prime \prime}$ from the center) which was also detected in the radio at $327 \mathrm{MHz}$ by Taylor et al. (1996) as WSRTGP2030+3840 (48" from the center). According to Taylor's criterion, it is a compact HII region. Its total IR luminosity corresponds to a B1 MS star at DM $=11^{\mathrm{m}}$. The CMD shows a poorly defined MS distribution centered on $A_{K}=2.5^{\mathrm{m}}$ with a high dispersion. From the PSC, we found a bright star $12^{\prime \prime}$ away from the cluster center with compatible reddening. Assuming $\mathrm{DM}=11^{\mathrm{m}}$, we have $M_{K}=-4.4^{\mathrm{m}}$ consistent with an $\mathrm{O} 5 \mathrm{~V}$ main sequence star, much earlier than the B1V type suggested by the IR observations. $\mathrm{DM}=10^{\mathrm{m}}$ would lead to $N_{\mathrm{OB}}=10 \pm 4, M_{\text {low }}=380 M_{\odot}$ and a central B0V star while $\mathrm{DM}=12^{\mathrm{m}}$ would lead to a O3V star, $N_{\mathrm{OB}}=50 \pm 21$ and $M_{\text {low }}=720 M_{\odot}$.

\section{$\mathrm{Cl} 08$}

The center of this cluster is very close to the HII region DR 15 (11" from center), IRAS 20306+4005 (24", also a maser) and numerous radio sources. According to Odenwald et al. (1990) and Comerón \& Torra (2001) the distance to DR15 is about $1 \mathrm{kpc}$, a value that we will adopt here. The CMD is very scattered and the morphology is confused because of two superimposed structures with different reddening and probably also different distances. From the PSC, we found a bright star 35" away from the cluster center with compatible reddening. Assuming $\mathrm{DM}=10^{\mathrm{m}}$, we have $M_{K}=-1.7^{\mathrm{m}}$ consistent with an B2V main sequence star. DM $=9^{\mathrm{m}}$ would lead to a B5V star, $N_{\mathrm{OB}}=13 \pm 5$ and $M_{\text {low }}=270 M_{\odot}$ while DM $=11^{\mathrm{m}}$ would lead to a B0.5V star, $N_{\mathrm{OB}}=25 \pm 10$ and $M_{\text {low }}=990 M_{\odot}$. Our results are compatible with the picture of a small nascent cluster between $\mathrm{DM}=10^{\mathrm{m}}$ and $11^{\mathrm{m}}$, powered by a B star, as proposed by Colley (1980).
This cluster appears as a small and concentrated structure, coincident with the HII region DR7 ( $38^{\prime \prime}$ from the center) and the corresponding IR source IRAS20264+4042 (30" from the center). The distance to DR7 is not well known. It was first estimated to be $1.5 \mathrm{kpc}$ (Campbell et al. 1982). Later, large radial velocities ruled out this first hypothesis and located DR7 in the Perseus arm at $7.5 \mathrm{kpc}$ (Piepenbrink \& Wendker 1988; Odenwald \& Schwartz 1993). More recently, Comerón \& Torra (2001) estimated a minimum of $3.6 \mathrm{kpc}$ from near infrared measurements, while Dutra \& Bica (2001) derived again a shorter estimate of $1.1 \mathrm{kpc}$ by fitting the upper MS in a colorcolor diagram using 2MASS data. The CMD is moderately scattered, most of the stars of the structure having reddening between $A_{K}=1.0^{\mathrm{m}}$ and $A_{K}=2.5^{\mathrm{m}}$. The morphology is a clear globular shape immediately surrounded by extremely obscured areas. It could correspond to a small nascent cluster or only to the core of a bigger structure. In the short distance hypothesis, assuming DM $=11^{\mathrm{m}}$, this cluster has no $\mathrm{O}$ star and the center coincides with a population of early B stars. The star that lies the closest to the cluster center with compatible reddening would have a spectral type of B1V. The total number of B stars in the cluster would amount to 22. This result corresponds to the description of Odenwald et al. (1986). With $\mathrm{DM}=14^{\mathrm{m}}$, the completeness is achieved only for B2 or earlier stars. We would have $N_{\mathrm{OB} 2}=44 \pm 15$, with $8 \pm 5 \mathrm{O}$ stars, $M_{\text {low }}=1910 M_{\odot}, M_{\text {up }}=4620 M_{\odot}$ and an O3V or giant type central star. This would lead to a very powerful HII region, as suggested by Wendker et al. (1991).

\section{$\mathrm{Cl} 10$}

This previously unknown cluster is located $2^{\circ}$ to the south east of the Cygnus OB2 association, in a less obscured region. We have not identified any possible counterpart for this cluster. Its characteristics are quite different from those of the preceding objects. Its CMD has a clear MS distribution centered on $A_{K}=1.0^{\mathrm{m}}$ with low scatter and a turn-off point near $\mathrm{B} 3 / \mathrm{B} 5$ type assuming our default value of $\mathrm{DM}=11^{\mathrm{m}}$. There are no O stars, few B stars but about 200 A to F stars having a clear globular morphology with a radius of $6.7^{\prime}$. The cluster seems quite massive and has an unusual steep IMF of $\Gamma=-1.98 \pm 0.25$. For DM $=10^{\mathrm{m}}$, the number of $\mathrm{B}$ stars drops to $5 \pm 2$ and $M_{\text {low }}=510 M_{\odot}$. For DM $=12^{\mathrm{m}}$ we have $N_{\mathrm{OB}}=113 \pm 44$ and $M_{\text {low }}=2190 M_{\odot}$. The absence of any HII region and the probable low turn-off suggest that this cluster could be quite evolved.

\section{$\mathrm{Cl} 11$}

This object lies $1^{\circ} 35^{\prime}$ to the east of the Cygnus OB2 association and is very close to many radio sources and to the HII region IRAS $20375+4109$ (2.4' from the center). The center of DR 22 is located $4.6^{\prime}$ to the west. Distance estimates are between $1 \mathrm{kpc}$ (Dutra \& Bica 2001) and $3 \mathrm{kpc}$ (Campbell et al. 1982). Odenwald et al. (1986) conclude that the ionizing flux is probably created by a single $\mathrm{O} 6 \mathrm{~V}$ star at $3 \mathrm{kpc}$. A more 
Table 5. Brightest star within $25 \%$ of the cluster radius with compatible reddening.

\begin{tabular}{|c|c|c|c|c|c|c|c|c|c|}
\hline Name & $\begin{array}{l}\alpha^{a} \\
(\mathrm{hms})\end{array}$ & $\begin{array}{l}\delta^{a} \\
(\mathrm{dms})\end{array}$ & $\begin{array}{l}\Delta^{b} \\
\left({ }^{\prime \prime}\right)\end{array}$ & $\begin{array}{l}J \\
\text { (mag) }\end{array}$ & $\begin{array}{l}K \\
\text { (mag) }\end{array}$ & $\begin{array}{l}A_{K}{ }^{c} \\
\text { (mag) }\end{array}$ & $\begin{array}{l}M_{K}{ }^{d} \\
\text { (mag) }\end{array}$ & Sp. Type ${ }^{e}$ & IR Sp. Type ${ }^{f}$ \\
\hline $\mathrm{Cl} 01$ & $20^{\mathrm{h}} 24^{\mathrm{m}} 28.6^{\mathrm{s}}$ & $+36^{\circ} 24^{\prime} 34.6^{\prime \prime}$ & 54 & 12.76 & 10.68 & 1.2 & -1.0 & $\mathrm{~B} 3 \mathrm{~V}$ & \\
\hline $\mathrm{Cl} 02$ & $20^{\mathrm{h}} 27^{\mathrm{m}} 26.8^{\mathrm{s}}$ & $+37^{\circ} 22^{\prime} 48.0^{\prime \prime}$ & 27 & 10.38 & 5.85 & 2.8 & -5.9 & * & $\mathrm{B} 0 \mathrm{~V}^{1}, \mathrm{~B} 1 \mathrm{~V}^{2}$ \\
\hline $\mathrm{Cl} 03$ & $20^{\mathrm{h}} 25^{\mathrm{m}} 37.4^{\mathrm{s}}$ & $+39^{\circ} 24^{\prime} 38.0^{\prime \prime}$ & 15 & 10.41 & 7.87 & 1.5 & -4.6 & $\mathrm{O} 4 \mathrm{~V}$ & \\
\hline $\mathrm{Cl} 04$ & $20^{\mathrm{h}} 29^{\mathrm{m}} 37.1^{\mathrm{s}}$ & $+39^{\circ} 01^{\prime} 56.0^{\prime \prime}$ & 16 & 12.56 & 8.70 & 2.4 & -4.7 & $\mathrm{O} 4 \mathrm{~V}$ & $09.5 \mathrm{~V}^{1}, \mathrm{~B} 1 \mathrm{~V}^{2}$ \\
\hline $\mathrm{Cl} 05$ & $20^{\mathrm{h}} 27^{\mathrm{m}} 10.6^{\mathrm{s}}$ & $+39^{\circ} 26^{\prime} 28.0^{\prime \prime}$ & 21 & 13.62 & 9.62 & 2.5 & -3.9 & $07.5 \mathrm{~V}$ & $\mathrm{~B} 0 \mathrm{~V}^{1,3}$ \\
\hline $\mathrm{Cl} 06$ & $20^{\mathrm{h}} 31^{\mathrm{m}} 45.5^{\mathrm{s}}$ & $+38^{\circ} 57^{\prime} 59.2^{\prime \prime}$ & 2 & 10.52 & 8.41 & 1.3 & -3.9 & $07.5 \mathrm{~V}$ & $\mathrm{~B} 0.5 \mathrm{~V}^{1}$ \\
\hline $\mathrm{Cl} 07$ & $20^{\mathrm{h}} 32^{\mathrm{m}} 27.0^{\mathrm{s}}$ & $+38^{\circ} 51^{\prime} 25.6^{\prime \prime}$ & 13 & 11.18 & 8.36 & 1.7 & -4.3 & $05.5 \mathrm{~V}$ & $\mathrm{~B} 1 \mathrm{~V}^{2}$ \\
\hline $\mathrm{Cl} 08$ & $20^{\mathrm{h}} 32^{\mathrm{m}} 31.0^{\mathrm{s}}$ & $+40^{\circ} 16^{\prime} 50.1^{\prime \prime}$ & 36 & 12.62 & 9.95 & 1.7 & -1.7 & $\mathrm{~B} 2 \mathrm{~V}$ & $\mathrm{~B} 0 \mathrm{~V}^{1}, \mathrm{~B} 1 \mathrm{~V}^{2}$ \\
\hline $\mathrm{Cl} 09$ & $20^{\mathrm{h}} 28^{\mathrm{m}} 11.4^{\mathrm{s}}$ & $+40^{\circ} 51^{\prime} 21.0^{\prime \prime}$ & 34 & 12.46 & 10.02 & 1.5 & -2.5 & B1V & $\mathrm{O} 9.5 \mathrm{~V}^{1}, \mathrm{~B} 0 \mathrm{~V}^{3}, \mathrm{~B} 1 \mathrm{~V}^{2}$ \\
\hline $\mathrm{Cl} 10$ & $20^{\mathrm{h}} 39^{\mathrm{m}} 13.3^{\mathrm{s}}$ & $+39^{\circ} 58^{\prime} 15.8^{\prime \prime}$ & 21 & 11.31 & 9.43 & 1.1 & -2.7 & $\mathrm{~B} 0.5 \mathrm{~V}$ & \\
\hline $\mathrm{Cl} 11$ & $20^{\mathrm{h}} 39^{\mathrm{m}} 34.1^{\mathrm{s}}$ & $+41^{\circ} 16^{\prime} 56.5^{\prime \prime}$ & 100 & 10.08 & 8.14 & 1.1 & -4.0 & $\mathrm{O} 7 \mathrm{~V}$ & $\mathrm{O}^{8} \mathrm{~V}^{4}, 09 \mathrm{~V}^{1,3}$ \\
\hline $\mathrm{Cl} 12$ & $20^{\mathrm{h}} 35^{\mathrm{m}} 23.0^{\mathrm{s}}$ & $+42^{\circ} 22^{\prime} 02.4^{\prime \prime}$ & 33 & 9.42 & 8.16 & 0.9 & -3.7 & $08.5 \mathrm{~V}$ & $\mathrm{O} 7 \mathrm{~V}^{4}$ \\
\hline $\mathrm{Cl} 13$ & $20^{\mathrm{h}} 38^{\mathrm{m}} 29.1^{\mathrm{s}}$ & $+42^{\circ} 06^{\prime} 18.9^{\prime \prime}$ & 6 & 10.70 & 9.14 & 0.9 & -2.8 & B $0.5 \mathrm{~V}$ & $08.5 \mathrm{~V}^{4}, 09.5 \mathrm{~V}^{1}$ \\
\hline $\mathrm{Cl} 14$ & $20^{\mathrm{h}} 35^{\mathrm{m}} 43.0^{\mathrm{s}}$ & $+42^{\circ} 29^{\prime} 41.9^{\prime \prime}$ & 18 & 8.22 & 6.99 & 0.7 & -4.2 & $05.5 \mathrm{~V}$ & \\
\hline $\mathrm{Cl} 15$ & $20^{\mathrm{h}} 40^{\mathrm{m}} 29.8^{\mathrm{s}}$ & $+42^{\circ} 03^{\prime} 13.7^{\prime \prime}$ & 87 & 9.24 & 7.62 & 1.0 & -4.3 & $\mathrm{O} 5 \mathrm{~V}$ & $\mathrm{O} 4 \mathrm{~V}^{4}$ \\
\hline
\end{tabular}

${ }^{a} \mathrm{~J} 2000$, from the 2MASS PSC.

${ }^{b}$ Angular distance to the estimated cluster center.

${ }^{c}$ Absorption in the $\mathrm{K}$ band estimated from Eq. (5).

${ }^{d}$ Assuming the DM quoted in Table 4.

${ }^{e}$ Assuming a luminosity class of $V$ and the DM quoted in Table 4.

${ }^{f}$ Spectral type derived from the far IR luminosity of the corresponding HII region and the DM quoted in Table 4.

${ }^{1}$ Harris \& Cl egg (1982), ${ }^{2}$ Odenwald \& Schwarz (1993), ${ }^{3}$ Odenwald et al. (1986), ${ }^{4}$ Campbell et al. (1982).

${ }^{*} K$ magnitude from 2MASS do not correspond to a single MS star at DM $=9$.

recent work of the same author (Odenwald 1993) considers a distance of $2 \mathrm{kpc}$ and associates DR22 with the Cygnus OB2 complex. We choose here $\mathrm{DM}=11^{\mathrm{m}}$ as a mean value. $\mathrm{Cl} 11$ is the most massive cluster studied in this work. It has a rather scattered CMD with $A_{K}=1.0^{\mathrm{m}}$ to $A_{K}=2.5^{\mathrm{m}}$. The morphology is clearly globular, quite extended and shows a dense core. The IMF slope has an unusually steep value of $\Gamma=-1.82 \pm 0.25$. From the PSC, we found a bright star $1.7^{\prime}$ away from the cluster center with compatible reddening. Assuming DM $=11^{\mathrm{m}}$, we have $M_{K}=-4.0^{\mathrm{m}}$ consistent with an $\mathrm{O} 7 \mathrm{~V}$ star. $\mathrm{DM}=10^{\mathrm{m}}$ would lead to a B0V star, $N_{\mathrm{OB}}=29 \pm 7$ and $M_{\text {low }}=980 M_{\odot}$ while $\mathrm{DM}=12^{\mathrm{m}}$ would lead to $\mathrm{O} 3 \mathrm{~V}$ or a giant class star, $N_{\mathrm{OB}}=203 \pm 47$ and $M_{\text {low }}=4760 M_{\odot}$. Our analysis is coherent with the picture of a massive young cluster associated with Cygnus OB2 and probably powered by an 06/7V type central star.

\section{$\mathrm{Cl} 12$}

This object lies $1^{\circ} 16^{\prime}$ to the northeast of the Cygnus OB2 association. The nearest objects are the X-ray source AX J2035.4+4222 (48"), the HII regions [L89b]81.253+01.123 (4') and DR17 (4.2'). Cl 12 and $\mathrm{Cl} 14$ (see below) are apparently close to each other $\left(9.5^{\prime}\right)$, but do not correspond to the same absorption range. $\mathrm{Cl} 12$ corresponds to stars having $A_{K}=1.0^{\mathrm{m}}$ to $2.0^{\mathrm{m}}$, while $\mathrm{Cl} 14$ is less obscured. Distance estimates for DR17 are comprised between $1.1 \mathrm{kpc}$ (Dutra \& Bica 2001) and $3 \mathrm{kpc}$ (Campbell et al. 1982).
We chose $\mathrm{DM}=11^{\mathrm{m}}$ as a mean value for $\mathrm{Cl} 12$. This cluster is quite massive and has a clearly globular morphology. From the PSC, we found a bright star with compatible reddening 1.9' away from the cluster center. Assuming $\mathrm{DM}=11^{\mathrm{m}}$, we have $M_{K}=-3.8^{\mathrm{m}}$ consistent with an O8V star. DM $=10^{\mathrm{m}}$ would lead to a B0.5V star, $N_{\mathrm{OB}}=13 \pm 3$ and $M_{\text {low }}=300 M_{\odot}$ while $\mathrm{DM}=12^{\mathrm{m}}$ would lead to a O3V star, $N_{\mathrm{OB}}=80 \pm 17$ and $M_{\text {low }}=1350 M_{\odot}$. Our results fit well with the picture of a young cluster associated to Cygnus OB2, with probable DM between $10.5^{\mathrm{m}}$ and $11.5^{\mathrm{m}}$.

\section{$\mathrm{Cl} 13$}

This is a very small, dense and obscured cluster located near the radio source WSRTGP 2036+4155 (12"), the IR source RAFGL $2620\left(3^{\prime}\right)$ and the HII region [L89b]81.480+00.561 $\left(6^{\prime}\right)$. Note that the center of the radio complex W75/DR21 is situated $15.6^{\prime}$ to the northeast. The DR21 radio complex has been located at $3 \mathrm{kpc}$ by Campbell (1982) and by Piepenbrink \& Wendker (1988). Odenwald (1993) favored a shorter estimate of 1.5 to $2 \mathrm{kpc}$. Assuming $\mathrm{DM}=11^{\mathrm{m}}$, we count only $12 \mathrm{~B}$ stars and no O stars. A very bright foreground star lies so close to the cluster center that the star population and size may be underestimated despite our corrections. From the PSC, we found a bright star 4" away from the cluster center with compatible reddening. Assuming $\mathrm{DM}=11^{\mathrm{m}}$, we have $M_{K}=-1.9^{\mathrm{m}}$ consistent with a B1.5V star. $\mathrm{DM}=10^{\mathrm{m}}$ would lead to a B3V star, $N_{\mathrm{OB}}=1 \pm 1$ and 
$M_{\text {low }}=160 M_{\odot}$ while DM $=12^{\mathrm{m}}$ would lead to a B0.5V star, $N_{\mathrm{OB}}=36 \pm 24$ and $M_{\text {low }}=440 M_{\odot}$. Our results fit well into the picture of a small nascent cluster powered by a central early B star at DM between $11^{\mathrm{m}}$ and $12^{\mathrm{m}}$.

\section{$\mathrm{Cl} 14$}

This object lies $12.2^{\prime}$ to the northeast of DR17 and is very close to $\mathrm{Cl} 12$. The nearest objects are the $\mathrm{X}$-ray source AX J2035.4+4229 (3'), two small radio sources and the IR source HFE67. The nearest HII region are [L89b]81.342+01.123 $\left(8^{\prime}\right)$ and DR17 $\left(9^{\prime}\right)$. This object is very close to $\mathrm{Cl} 12$, which was supposed to be at DM $=11^{\mathrm{m}}$. Yet it has a much lower absorption which lead us to favor a slightly lower distance estimate of DM $=10.5^{\mathrm{m}}$. Under this assumption, we count only $12 \mathrm{~B}$ stars and one $\mathrm{O}$ star. The CMD shows a MS-like distribution centered on $A_{K}=1.0$. From the PSC, we found a bright star $18^{\prime \prime}$ away from the cluster center and with compatible reddening. Assuming $\mathrm{DM}=10.5^{\mathrm{m}}$, we have $M_{K}=-4.2^{\mathrm{m}}$ consistent with a $05.5 \mathrm{~V}$ star. DM $=9.5^{\mathrm{m}}$ would lead to a B0V star, $N_{\mathrm{OB}}=8 \pm 3$ and $M_{\text {low }}=200 M_{\odot}$ while $\mathrm{DM}=11.5^{\mathrm{m}}$ would lead to super giant $\mathrm{O}$ star, $N_{\mathrm{OB}}=41 \pm 14$ and $M_{\text {low }}=850 M_{\odot} . \mathrm{Cl} 14$ is very similar to $\mathrm{Cl} 03$ and the same discussion applies. It is probably an evolved and quite close object, with an coincident foreground bright star near the center.

\section{$\mathrm{Cl} 15$}

This object is located $10.8^{\prime}$ to the northwest of DR23. The nearest object is the radio source MITG J2040+4200 $\left(1^{\prime}\right)$. DR23 has been located at $3 \mathrm{kpc}$ by Campbell (1982). Odenwald (1993) favored a shorter estimate of 1.5 to $2 \mathrm{kpc}$. $\mathrm{Cl} 15$ has a very scattered CMD with $A_{K}=1.0^{\mathrm{m}}$ to $A_{K}=2.5^{\mathrm{m}}$ but a clearly globular morphology with a rather large radius of $6.9^{\prime}$. Assuming $\mathrm{DM}=11^{\mathrm{m}}$ we have $N_{\mathrm{OB}}=62 \pm 20$ and $M_{\text {low }}=720 M_{\odot}$ From the PSC, we found a very bright star $87^{\prime \prime}$ away from the cluster center with compatible reddening. It has $M_{K}=-4.3^{\mathrm{m}}$ consistent with a O5V star. DM $=10^{\mathrm{m}}$ would lead to a B0V star, $N_{\mathrm{OB}}=29 \pm 9$ and $M_{\text {low }}=370 M_{\odot}$ while $\mathrm{DM}=12^{\mathrm{m}}$ would lead to giant type star, $N_{\mathrm{OB}}=90 \pm 30$ and $M_{\text {low }}=1513 M_{\odot}$. Our results fit well into the picture of a powerful young cluster associated with the DR23 radio complex and powered by a central early $\mathrm{O}$ star at DM between $11^{\mathrm{m}}$ and $12^{\mathrm{m}}$.

\section{Discussion}

\subsection{Classification of new cluster candidates}

The new cluster candidates we studied in this work can be divided into 3 classes. $\mathrm{Cl} 01, \mathrm{Cl} 03, \mathrm{Cl} 10$ are quite extended, previously unknown clusters, with no known counterpart or coincident HII region and moderate reddening. They could be quite evolved and moderately massive objects $\left(M_{\text {low }} \approx 1000 M_{\odot}\right)$ in the local spiral arm. Yet, they could also be more distant and massive clusters located in the Perseus arm and seen through holes in the absorption pattern. Although less massive, $\mathrm{Cl} 14$ also fits well into this category.
All the other cluster candidates have a coincident HII region. They have a high central star density and a heavy absorption with $A_{V}$ between $10^{\mathrm{m}}$ and $30^{\mathrm{m}}$. All are possibly powered by early $\mathrm{B}$ or $\mathrm{O}$ stars. These clusters are probably very young objects.

The second class is comprised of $\mathrm{Cl} 02, \mathrm{Cl} 04, \mathrm{Cl} 05, \mathrm{Cl} 07$, $\mathrm{Cl} 08, \mathrm{Cl} 12$ and $\mathrm{Cl} 13$, which are are quite small with a typical radius of $2^{\prime}$. They have a minimum mass of about 400 to $500 M_{\odot}$. DM $=10.5^{\mathrm{m}}$ to $11.5^{\mathrm{m}}$ seems a consistent distance range and locates these clusters in the local arm, except for $\mathrm{Cl} 02$ that probably lies closer $(\mathrm{DM}=9)$.

The third class is formed by $\mathrm{Cl} 06$ and $\mathrm{Cl} 15$, which are more extended structures probably located in the local arm. They have a typical radius of about $5^{\prime}$ and a minimum mass of about $1000 M_{\odot} . \mathrm{Cl} 11$ also belongs to this category but is more massive. It has a minimum mass of $2000 M_{\odot}$ and an important OB population. It could then be of the Orion A class.

For $\mathrm{Cl} 09$, the distance estimate is particularily uncertain. If we locate this object in the local spiral arm, it clearly belongs to the second category. On the other hand, assuming that it is located in the Perseus arm, as suggested by the high radial velocity of its possibly associated HII region DR7 (Wendker et al. 1991), it belongs to the third category. In this case we would have another Orion A class cluster or one even more powerful.

\subsection{Spectral type disagreement}

The earliest spectral type as determined from the 2MASS data and the IR luminosities of associated HII regions shows a good agreement in 6 cases $(\mathrm{Cl} 08, \mathrm{Cl} 09, \mathrm{Cl} 11, \mathrm{Cl} 12, \mathrm{Cl} 13, \mathrm{Cl} 15)$, while in 5 cases $(\mathrm{Cl} 02, \mathrm{Cl} 04, \mathrm{Cl} 05, \mathrm{Cl} 06, \mathrm{Cl} 07)$, IR measurements suggest a considerably later spectral type (see Table 5). As an example, for $\mathrm{Cl} 04\left(A_{K}=2.4^{\mathrm{m}}\right)$, IR luminosities suggest a mean spectral type of $\mathrm{B} 0 \mathrm{~V}$, while we obtain an absolute $K$ magnitude of $-4.7^{\mathrm{m}}$. If the star would indeed be of spectral type $\mathrm{B} 0 \mathrm{~V}$, an absolute $K$ magnitude of $-3.4^{\mathrm{m}}$ would be expected (see Appendix of Paper I), 1.3 mag fainter than our finding. Excepting the case of $\mathrm{Cl} 02$, the discrepancy in the absolute $K$ magnitude varies from 0.45 to $1.8^{\mathrm{m}}$. Several reasons may explain this disagreement.

Firstly, the star we selected from the 2MASS database as the brightest cluster member may be simply a foreground star. We estimated the probability of a chance coincidence by calculating the density of stars brighter than our tentatively assigned brightest member in a large field of $4^{\circ} \times 4^{\circ}$ around the cluster, considering only objects with reddenings within the cluster interval (see Table 4). Multiplication with the area that we searched for the brightest member (25\% of the cluster radius) gives the expected number of field stars, which we convert using the Poisson law into the probability of observing at least one such star within the searched area. In this way we determine a typical chance coincidence probability of a few percents for most of the clusters. Exceptions are $\mathrm{Cl} 06, \mathrm{Cl} 08$ and $\mathrm{Cl} 15$ for which we find a chance coincidence probabilities of $12 \%$, $24 \%$ and $9 \%$, respectively. Hence, we cannot exclude that the brightest member we selected is in some cases indeed a foreground object. However, it is only in the case of $\mathrm{Cl} 08$ where 
the chance coincidence probability is sufficently high to possibly explain the discrepancy between ours and the IR spectral type assignement.

Secondly, the selected star may be an evolved giant instead of a main-sequence object leading to a different luminosity spectral-type calibration relation. However, IR estimates suffer from the same problem (they all assume a luminosity class V), and by comparing the $U V$ luminosity estimates of the atmosphere models of Schaerer \& de Koter (1997) to the $K$ magnitudes calibrations (Wegner 1994; Vacca et al. 1996) for various luminosity classes we find that in both cases, the $U V$ and $K$ luminosity should vary by about the same factor. Thus, even if we misclassified the star in luminosity class, we should find about the same spectral type with both approaches if it is the selected star that powers the associated HII region.

Thirdly, the 2MASS magnitudes could be simply unreliable, either due to source confusion in the dense central cluster regions, or due to the possible presence of diffuse nebular emission. The very large discrepancy noted for $\mathrm{Cl} 02$ (about $3 \mathrm{mag}$ ), for which we suggest the central star of the bipolar emission nebula S106 as brightest cluster member, can probably be explained this way. Although the 2MASS PSC quality flags signal a reliable magnitude for this star, the presence of the bipolar jet may lead to a considerable overestimation of the star's magnitude (Bally et al. 1998).

Fianlly, we note that the spectral type mismatch seems to increase with increasing reddening. Thus, if for some reason we overestimated the reddening $A_{K}$, the apparent mismatch may be explained. In particular, we used Eq. (5) to estimate the reddening which relies on a normal reddening slope of $R_{K}=0.66$ and the assumption of having a main-sequence star. It is well known, however, that $R_{K}$ may vary in star forming regions (Mathis 1990), hence our $A_{K}$ estimate probably suffers from substantial uncertainty (this point is not very relevant for our stellar selection since in general we employ relatively large $A_{K}$ intervals; see Table 4). On the other hand, the IR luminosity estimate is based on the assumption that all ionizing flux is reradiated in the infrared domain (Odenwald 1989). If however for some reason only a fraction of the $U V$ flux is converted into IR radiation in the heavily obscured clusters, IR observations should lead to an underestimation of the stellar luminosity - as suggested by our analysis. Thus, the two estimates we quote in Table 5 are probably boundaries to the real earliest spectral type in the cluster, which is probably situated somewhere between these limits.

\subsection{Mass segregation}

During the analysis of radial star density profiles for our cluster candidates, we recognized a clear trend of smaller cluster radius with increasing stellar mass. To illustrate this finding, we determined the half population radius (which turns out to be the most stable cluster dimension parameter with respect to details of the stellar selections) for 3 non-overlapping stellar mass intervals (G0-F3, F2-A0, and B9-O3). The results are summarized in Table 6. A dash indicates that severe incompleteness hampered the determination of the half population radius for
Table 6. Evolution of half population radius with spectral range in units of arcmin.

\begin{tabular}{llll}
\hline \hline Name & {$[G 0$ F3] } & {$[$ F2 A0] } & {$[$ [B9 O3] } \\
\hline IC 4996 & - & 2.4 & 1.0 \\
VdB 130 & - & 4.8 & 5.9 \\
Ber 85 & 3.4 & 2.6 & - \\
Ber 86 & 3.7 & 2.7 & 2.3 \\
Ber 87 & - & - & 4.1 \\
NGC 6913 & 5.3 & 4.6 & 2.0 \\
NGC 6910 & 5.5 & 3.9 & 2.0 \\
Cl 01 & - & 1.8 & 1.2 \\
Cl 02 & - & 0.8 & 0.4 \\
Cl 03 & - & 2.6 & 0.9 \\
Cl 04 & - & - & 0.5 \\
Cl 05 & - & - & 0.6 \\
Cl 06 & - & - & 1.0 \\
Cl 07 & - & - & 1.7 \\
Cl 08 & - & - & 0.5 \\
Cl 09 & - & 0.7 & 2.4 \\
Cl 10 & - & 7.1 & 2.3 \\
Cl 11 & - & 2.4 & 1.7 \\
Cl 12 & - & 1.4 & 0.8 \\
Cl 13 & - & 0.8 & 0.5 \\
Cl 14 & - & - & 0.5 \\
Cl 15 & - & 2.8 & 2.2 \\
\hline
\end{tabular}

the corresponding mass interval. We estimate the uncertainty of the radius determination to typically $\pm 0.2^{\prime}$.

The decrease of cluster radius with increasing mass (or earlier spectral types) is striking. Except of 2 clusters (VdB 130 and $\mathrm{Cl}$ 09), all objects show a more or less pronounced reduction of the half population radius between the spectral type intervals F2-A0 and B9-O3. For the 4 clusters for which we also have a radius estimate for the G0-F3 interval, the trend continues even to these small masses. Excluding VdB 130 and $\mathrm{Cl} 09$, we find an average ratio between the half population radius in the B9-O3 and F2-A0 interval of $0.56 \pm 0.17$, thus OB stars appear almost by a factor of 2 more concentrated than stars in the F2-A0 interval.

Our analysis is not the first that suggests mass segregation in young compact open clusters (see de Grijs et al. 2002 and references therein), yet the systematic finding of mass segregation in 13 out of 15 clusters provides an unprecedented large sample. It is surprising to find such an important mass segregation in such young clusters. Age estimates in the literature for the known clusters indicate that they all should be younger than $15 \mathrm{Myr}$, and our new cluster candidates that are most embedded in their natal molecular cloud are probably even younger. Due to their youth, it seems difficult to explain the observations by a dynamic segregation (the age being smaller than the typical relaxation time-scales), supporting the idea that primordial mass segregation may play an important role in cluster formation (Bonnell et al. 2001).

What is so special about $\mathrm{VdB} 130$ and $\mathrm{Cl} 09$ which show massive stars less concentrated than low-mass stars? VdB 130 shows only a slightly larger radius for B9-O3 than for F2-A9 stars which is possibly explained by confusion with massive 
stars from the surrounding $\mathrm{Cyg}$ OB1 association. For $\mathrm{Cl} 09$, the sample in the F2-A9 interval is very poor and we believe that the $0.7^{\prime}$ radius is probably underestimated. In addition, $\mathrm{Cl} 09$ lies close to Cyg OB2 and we cannot exclude a contamination by this large star cluster. Therefore, it may be that VdB 130 and $\mathrm{Cl} 09$ do not show any mass segregation at all, and the results in Table 6 reflect rather the limits of our analysis in crowded, obscured areas.

\section{Conclusion}

We characterized 22 open clusters in the Cygnus area. For the previously known clusters, our results are in good agreement with previous works, validating our approach. 3 previously unknown, probably evolved clusters were discovered. Thanks to infrared data and reddening independent stellar selections, 12 clusters that spatially coincide with compact HII regions could be analyzed despite a heavy and variable obscuration, varying from $A_{V}=5^{\mathrm{m}}$ to $A_{V}=30^{\mathrm{m}}$. The clusters have a rather uniform IMF slope compatible with the Salpeter value of -1.35 .

According to our study and the derived characteristics, most of the DR HII regions of the Cygnus area host a very young or even nascent cluster. Except for $\mathrm{Cl} 02$ and perhaps $\mathrm{Cl} 09, \mathrm{DM}=10.5^{\mathrm{m}}$ to $11.5^{\mathrm{m}}$ seems a reasonable distance range. Furthermore, their spatial distribution shows that most of them are located at the edge of the compact association Cygnus OB2. Hence, our work supports the idea that this very powerful star concentration has triggered recent star formation events in the molecular clouds lying at its border.

Our analysis improves the knowledge of the massive star population distribution in the Cygnus area. It confirms that a large fraction of the young open clusters is hidden in dense interstellar clouds. The total OB star population in the seven known and less obscured clusters is $155 \pm 35$ while in the 15 new cluster candidates we count a total of $454 \pm$ 90 OB stars, about three times more. However, the total OB star population in the clusters of the Cygnus region represents only $25 \%$ of the OB population in the compact association Cygnus OB2 (see Paper I). This illustrates that such a supermassive object remains unique in the area.

The detailed analysis of the radial profiles reveals a clear tendency of mass segregation, which is observed in all except two clusters. This observation considerably increases the number of galactic young star clusters for which mass segregation is suggested (Grijs et al. 2002), and indicates that primordial mass segregation may be a common feature in young embedded clusters. In this context we want to mention that no mass segregation has been observed in the massive Cyg OB2 cluster for which we performed a similar analysis (Paper I). Possibly, the mode of cluster formation differs between such huge systems as Cyg OB2 and the small embedded clusters we studied in this work, which leaves a different imprint on the mass distribution that may inform us about the process of formation.

Finally, we note that we have no reliable distance information for most of the new cluster candidates we discussed in this paper, and our estimates rely mostly on possible correlations with HII regions (which have rather large distance uncertainties themselves) and the assumption that they lie probably in the local spiral arm which is situated between 1-2 kpc in the Cygnus region. Spectroscopic data is urgently needed to refine the spectral classification of the brightest member stars, which would help then to produce more reliable mass and population estimates for these interesting objects.

Acknowledgements. This publication make use of data products from the Two Micron All Sky Survey, which is a joint project of the University of Massachusets and the Infrared Processing and Analysis Center/California Institute of Technology, funded by the National Aeronautics and Space Administration and the National Science Foundation.

This research has also made use of the SIMBAD database, operated at CDS, Strasbourg, France and the WEBDA database, compiled by Jean-Claude Mermillod, Institute of Astronomy of the University of Lausanne, Switzerland.

\section{References}

Bally, J., \& Predmore, R. 1983, ApJ, 265, 778

Bally, J., Yu, K. C., Rayner, J., \& Zinnecker, H. 1998, AJ, 116, 1868

Barsony, M., Scoville, N. Z., Bally, J., \& Claussen, M. J. 1983, ApJ, 265, 778

Bonnell, I. A., Bate, M. R., Clarke, C. J., \& Pringle, J. E. 2001, MNRAS, 323, 785

Bruch, A., \& Sanders, W. L. 1983, A\&A, 121, 237

Campbell, M. F., Hoffman, W. F., Thronson, H. A., et al. 1982, AJ, 261,550

Casoli, F., Dupruz, C., Guerin, M., Combes, F., \& Boulanger, F. 1986, A\&A, 169, 281

Colley, D. 1980, MNRAS, 192, 377

Comerón, F., \& Torra, J. 1999, A\&A, 349, 605

Comerón, F., \& Torra, J. 1999, A\&A, 375, 539

Crawford, J. L., \& Barnes, J. V. 1977, AJ, 82, 606

Deeg, H. J., \& Ninkov, Z. 1996, A\&A, 119, 221

de Grijs, R., Johnson, R. A., Gilmore, G. F., \& Frayn, C. M. 2002, MNRAS, 331, 228

Delgado, A. J., \& Alfaro, E. J. 1998, AJ, 113, 713

Dickel, H. R., \& Wendker, H. J. 1978, A\&A, 66, 289

Dolidze, M. V. 1961, Astron. Cir., 223, 11-12

Dutra, C. M., \& Bica, E. 2001, A\&A, 376, 434

Garmany, C. D., \& Stencel, R. E. 1992, A\&AS, 94, 211

Harris, S., \& Clegg, P. E. 1982, MNRAS, 203, 965

Hoare, M. G., \& Muxlow, T. B. 1996, Radio Emission from the Stars and the Sun, ed. A. R. Taylor, \& J. M. Pavedes (San Francisco: ASP), ASP Conf. Ser., 93

King, I. 1962, AJ, 67, 471

Knödlseder, J. 2000, A\&A, 360, 539

Knödlseder, J., Cervino, M., Le Duigou, J. M., Meynet, G., et al. 2002, submitted to A\&A

Kroupa, P. 2001, MNRAS, 322, 231

Kurtz, S., Churchwell, E., \& Wood, D. O. S. 1994, AJS, 184, 3013

Lockman, F. J. 1990, ApJS, 71, 469

Loushin, R., Crutcher, R. M., \& Bieging, J. H. 1990, ApJ, 362, L67

Massey, P., Johnson, K. E., \& Degioia-Eastwood, K. 1995, AJ, 454, 151

Mathis, J. S. 1990, ARA\&A, 28, 37

Odenwald, S. F., Shivanandan, K., Campbell, M., et al. 1986, AJ, 306, 122

Odenwald, S. F. 1989, AJ, 97, 801 
Odenwald, S. F., Campbell, M. F., Shivanandan, K., et al. 1990, AJ, Taylor, D. G., Goss, W. M., \& Coleman, P. H. 1996, AJS, 107, 239 99,288

Odenwald, S. F., \& Schwartz, P. R. 1993, ApJ, 405, 706

Piepenbrink, A., \& Wendker, H. J. 1988, A\&A, 191, 313

Racine, R. 1974, AJ, 79, 945

Rieke, G. H., \& Lebofsky, M. J. 1985, ApJ, 288, 618

Schaerer, D., \& de Koter, A. 1997, A\&A, 322, 598

Turner, A. R., \& Forbes, D. 1982, PASP, 94, 789

Vacca, W. D., Garmany, C. D., \& Shull, J. M. 1996, ApJ, 460, 914

Vansevicius, V., Bridzius, A., Pucinskas, A., \& Sasaki, T. 1996, Baltic Astron., 5, 539

Wang, J. J., \& Hu, J. Y. 2000, A\&A, 356, 118

Wegner, W. 1994, MNRAS, 270, 229

Staude, H. J., Lenzen, R., Dyck, H. M., \& Schmidt, G. D. 1982, ApJ, 255,95

Wendkler, H. J., Higgs, L. A., \& Landecker, T. L. 1991, A\&A, 241, 551 